\title{
NIST Special Database 2 Structured Forms Database Version 2.0 Users' Guide
}

Darren Dimmick Michael Garris Charles Wilson

Patricia Flanagan

This publication is available free of charge from: https://doi.org/10.6028/NIST.NSRDS.2-2017 


\title{
NIST Special Database 2 Structured Forms Database Version 2.0 Users' Guide
}

\author{
Darren Dimmick \\ Michael Garris \\ Patricia Flanagan \\ Information Technology Laboratory \\ Information Access Division
}

March 2017

This publication is available free of charge from:

https://doi.org/10.6028/NIST.NSRDS.2-2017

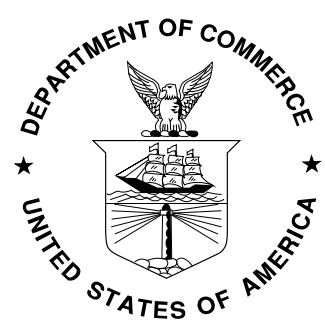

U.S. Department of Commerce Wilbur L. Ross, Jr., Secretary

National Institute of Standards and Technology Kenneth Rochford, Acting Under Secretary of Commerce for Standards and Technology and Director 


\section{Table of Contents}

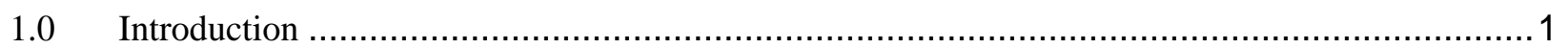

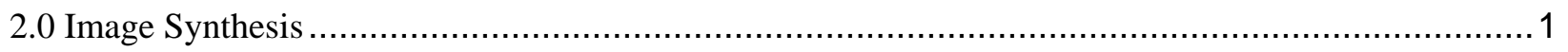

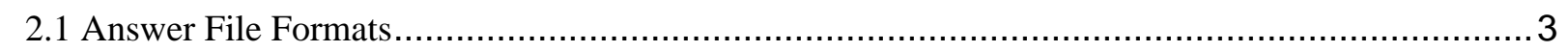

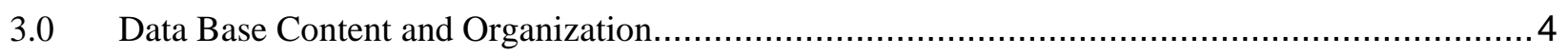

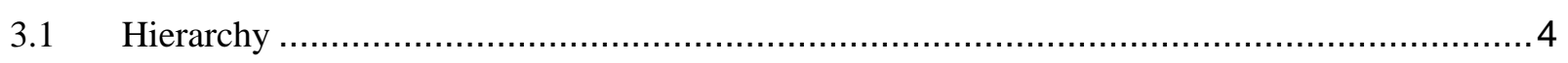

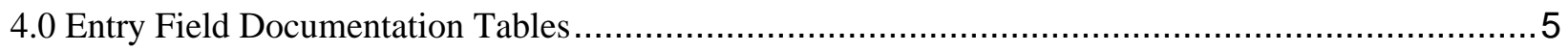

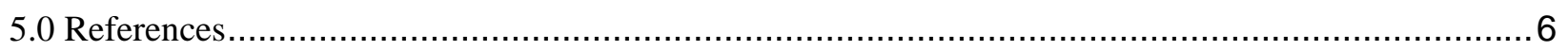

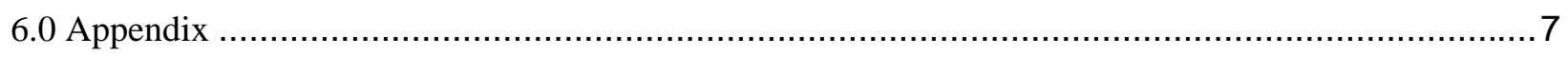

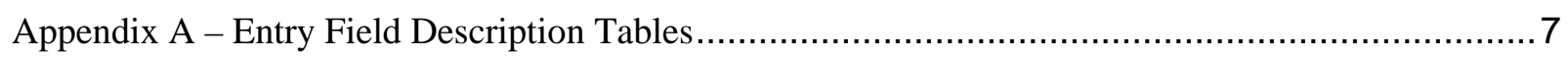




\subsection{Introduction}

This document describes the updated web release of the NIST Special Database 2, which contains 5590 PNG[1] synthesized completed IRS 1040 forms from 1988. The previously released CD-ROM NIST Special Database 2 consists of NIST IHEAD formatted images using a CCITT Group 4 compression[2] stored in binary black and white raster format. Databases of this magnitude are necessary to further the research and development of automated document processing systems.

This database is being distributed as a reference data set to be used by developers of document recognition and data capture systems to test and report results on a common corpus of images derived from structured forms containing machine printed data. The structured forms used in this database are 12 different tax forms from the IRS 1040 Package $X$ for the year 1988. These include Forms 1040, 2106, 2441, 4562, and 6251 together with Schedules A, B, C, D, E, F, and SE. Eight of these forms contain two pages or form faces making a total of 20 different form faces represented in the database.

The database contains 5590 full page images of completed tax forms. The images in this database appear to be real forms prepared by individuals but the images have been automatically derived and synthesized using a computer.

\subsection{Image Synthesis}

The entry field values on these forms have been automatically generated by a computer in order to make the data available without the danger of distributing privileged tax information. The computer derived entry field values are synthesized as images from one or more fonts of machine printed data. An image of an entry field value is produced by combining images of each character in the value. An entry field image is then inserted in a selected location within the corresponding field within a form image. The image data entered in a field in this way has been translated and rotated by small amounts to simulate effects of imperfect printing and imperfect alignment of a form in the printing device. Multiple examples of the digital representation of each character are used so that the pattern of the binary pixels representing each character is not consistently replicated but varies as it would in a sample of real tax forms. Both the form templates and the character examples are digitized at 300 pixels per inch. 


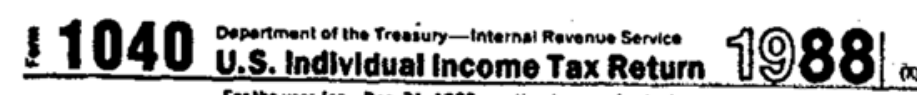

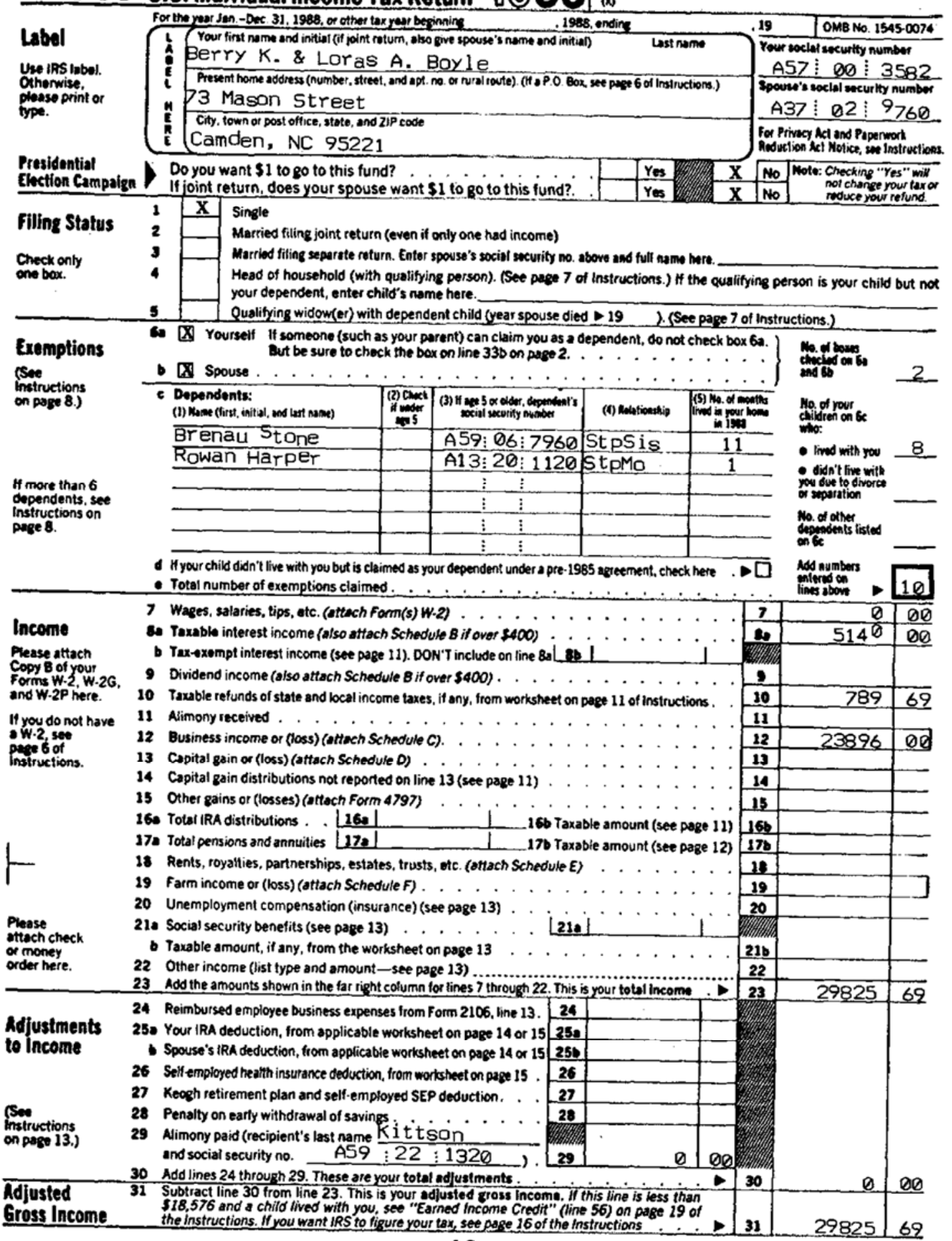
19

Figure 1 A representative image file of a completed form in NIST Special Database 2 


\subsection{Answer File Formats}

The values entered on the forms have been derived by a computer. These entry field values are stored separately from the image in an ASCII text file. This text file, one per completed structured form image, serves as an answer file which can be used to score the values hypothesized by a recognition system. The completed form's answer files from Figure 1 is listed in Figure 2. These text files are the ground truth against which recognition responses may be compared.

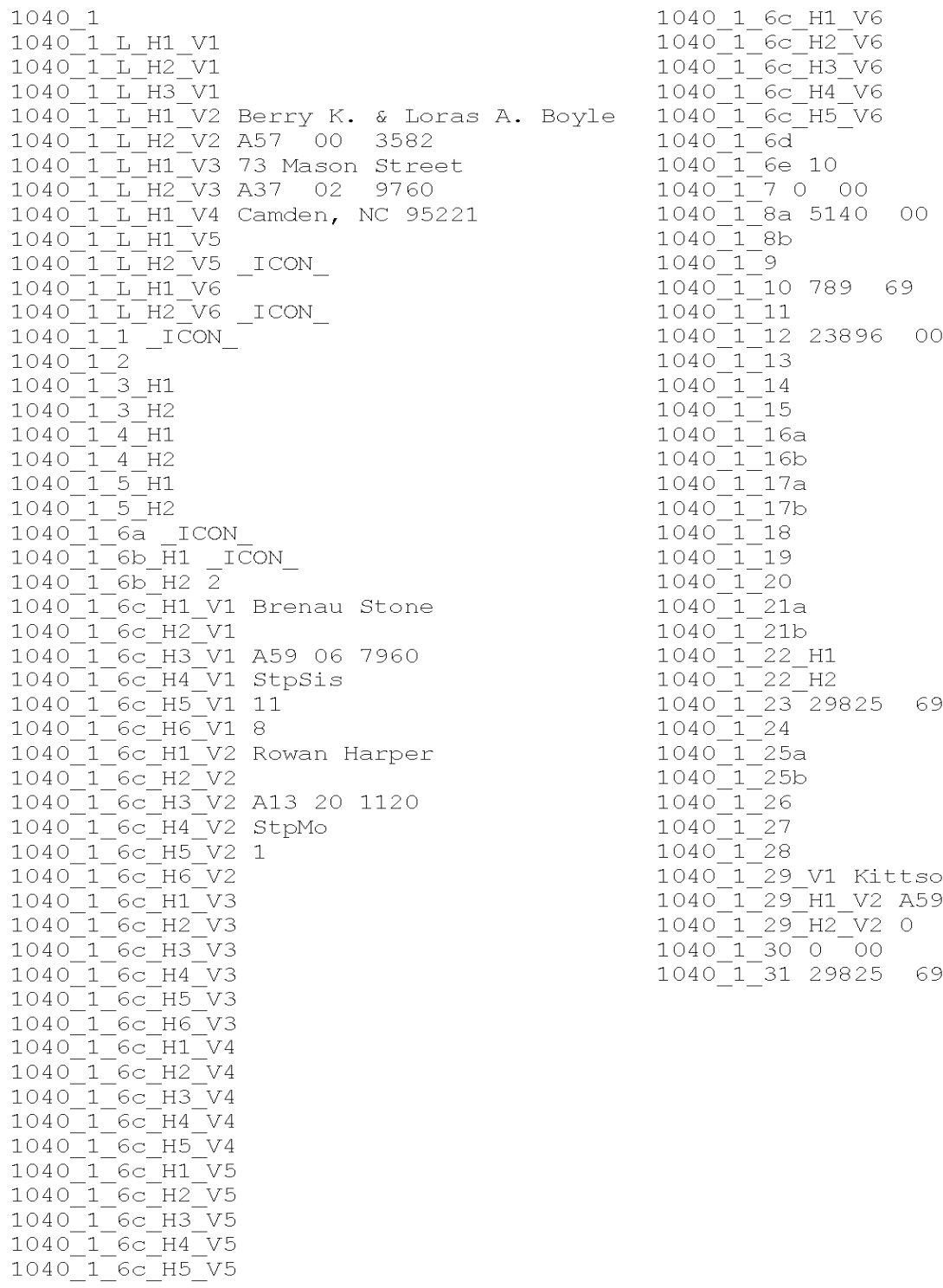

Figure 2: The answer file for the image shown in Figure 1. 
The first line in the text formatted file contains the identification of the form face in the referenced image. NIST Special Database 2 contains multiple form faces and therefore can be used for testing the forms identification ability of a document recognition system. The form type identification can be used to compute a system's accuracy in correctly identifying the form face contained in an image.

Each successive line in the answer file is an entry field identification and entry field value pair. The field identification string uniquely identifies which entry field is being referenced on a structured form. The field identifications used in this database are labeled on the form faces contained in Appendix A. The entry field value may be empty or it may contain a computer derived value. Empty entry field values model sparsely filled forms. An entry field value containing the token string "_ICON_" represents the existence of non-character information.

Examples of this type of information includes box check marks and signatures. Any other value listed for an entry field references the precise character information entered into the form image.

\subsection{Data Base Content and Organization}

NIST Special Database 2 contains 5590 full page images of completed structured forms in PNG format and correspondingly contains 5590 ASCII text answer files.

\subsection{Hierarchy}

There are 5590 full-page images of completed forms distributed in the data directory with 9 subdirectories within this database. The directory data has subdirectories sfrs_0, sfrs_1, through sfrs_8 each contain 100 synthesized tax submissions comprised of a random collection of completed form faces generated by a computer. Therefore, there are 900 total tax submissions in this database. Each submission is represented as a directory. An example of a submission directory $\mathbf{r 0 2 0 0}$ is illustrated in Figure 3. In this example, the directory sfrs_2 contains the 100 submission directories $\mathbf{r 0 2 0 0}$ through $\mathbf{r 0 2 9 9}$. The images associated with submission 200 are stored in the subdirectory r0200. There are on average 6.2 form images stored in a submission directory. In Figure 3, r0200 contains 9 synthesized form faces stored as the files r0200_00.png, r0200_01.png, through r0200_08.png where the last two digits in the file name uniquely index the form images. For each form face image, there is a corresponding answer file. The answer file for the image r0200_00.png is r0200_00.fmt, r0200_01.png is r0200_01.fmt, and so on. In this way, 5590 form images are stored in the database with their 5590 corresponding answer files accounting for 11180 individual files in all. 


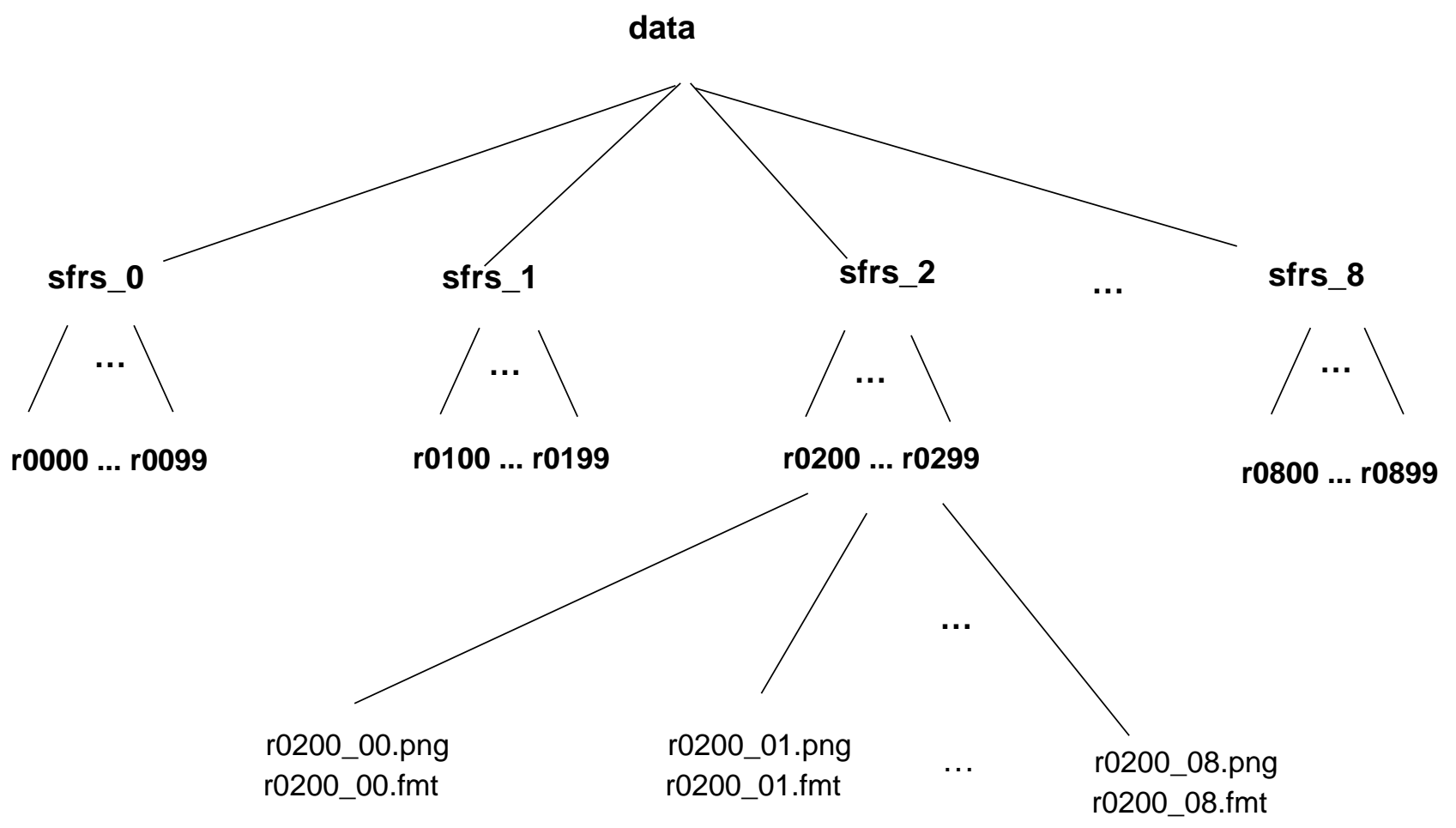

Figure 3. The file organization of the form images and answer files contained in NIST Special Database 2.

\subsection{Entry Field Documentation Tables}

The final set of information provided with this database is a collection of tables. These tables contain general knowledge about each entry field found on a structured form. This knowledge can be applied by system developers to guide the recognition process of their document processing system. These tables specify the data type and context associated with each entry field found on the form faces labeled in Appendix A. Formatted copies of these tables can be found in the directory tables within the top-level directory.

The directory contains 20 different tables, 1 for each of the 20 different form faces found in this database. Each line in these tables references a unique entry field from the corresponding form face. Entry fields are described by three columns of information. The first column in these tables contains entry field identifiers, the second column contains entry field data types, and the third column contains each entry field's associated context. Figures 4 and 5 list the possible entry field data types and contexts contained on the structured form faces used in this database. 


\begin{tabular}{|l|l|}
\hline \multicolumn{1}{|c|}{ TAG } & \multicolumn{1}{c|}{ DEFINITION } \\
\hline A, CA & Alphanumeric Field \\
F, FF, FP, FPER, & Floating Point Fields \\
FU & Integer Fields \\
I & Non-Character Fields (box markings, \\
ICON & signatures) \\
\hline
\end{tabular}

Figure. 4. The set of possible entry field data types.

\begin{tabular}{|l|l|}
\hline \multicolumn{1}{|c|}{ TAG } & \multicolumn{1}{c|}{ DEFINITION } \\
\hline DATA & Generic Data \\
\hline NAME & Names of People \\
\hline SSN & Social Security Numbers \\
\hline
\end{tabular}

Figure 5. The set of possible entry field contexts.

\subsection{References}

[1] "Information technology -- Computer graphics and image processing -- Portable Network Graphics (PNG): Functional specification," Internationa Organization for Standardization/International Electrotechnical Commission, ISO/IEC 15948:2004.

[2] "Facsimile Coding Schemes and Coding Control Functions for Group 4 Facsimile Apparatus, Fascicle VII.3 - Rec. T.6,” CCITT, 1984. 


\subsection{Appendix \\ Appendix A - Entry Field Description Tables}


Form 10401 Table A

\begin{tabular}{|c|c|c|}
\hline \multicolumn{3}{|c|}{ FORM_1040_1_Table_A } \\
\hline Entry Field & Type & Context \\
\hline 1040_1_L_H1_V1 & A & DATA \\
\hline 1040_1_L_H2_V1 & $A$ & DATA \\
\hline 1040_1_L_H3_V1 & A & DATA \\
\hline 1040_1_L_H1_V2 & A & NAME \\
\hline 1040_1_L_H2_V2 & A & SSN \\
\hline 1040_1_L_H1_V3 & A & DATA \\
\hline 1040_1_L_H2_V3 & $A$ & SSN \\
\hline 1040_1_L_H1_V4 & $A$ & DATA \\
\hline 1040_1_L_H1_V5 & ICON & DATA \\
\hline 1040_1_L_H2_V5 & ICON & DATA \\
\hline 1040_1_L_H1_V6 & ICON & DATA \\
\hline 1040_1_L_H2_V6 & ICON & DATA \\
\hline 1040_1_1 & ICON & DATA \\
\hline 1040_1_2 & ICON & DATA \\
\hline 1040_1_3_H1 & ICON & DATA \\
\hline 1040_1_3_H2 & A & NAME \\
\hline 1040_1_4_H1 & ICON & DATA \\
\hline 1040_1_4_H2 & A & NAME \\
\hline 1040_1_5_H1 & ICON & DATA \\
\hline 1040_1_5_H2 & $A$ & DATA \\
\hline 1040_1_6a & ICON & DATA \\
\hline 1040_1_6b_H1 & ICON & DATA \\
\hline 1040_1_6b_H2 & $\mathrm{I}$ & DATA \\
\hline 1040_1_6c_H1_V1 & $A$ & NAME \\
\hline 1040_1_6c_H2_V1 & ICON & DATA \\
\hline 1040_1_6c_H3_V1 & $A$ & SSN \\
\hline 1040_1_6c_H4_V1 & $A$ & DATA \\
\hline 1040_1_6c_H5_V1 & I & DATA \\
\hline 1040_1_6c_H6_V1 & $\mathrm{I}$ & DATA \\
\hline 1040_1_6c_H1_V2 & $A$ & NAME \\
\hline 1040_1_6c_H2_V2 & ICON & DATA \\
\hline 1040_1_6c_H3_V2 & $A$ & SSN \\
\hline 1040_1_6c_H4_V2 & A & DATA \\
\hline 1040_1_6c_H5_V2 & $\mathrm{I}$ & DATA \\
\hline FORM & & \\
\hline
\end{tabular}




\begin{tabular}{|c|c|c|}
\hline Entry Field & Type & Context \\
\hline 1040_1_6c_H6_V2 & $\mathrm{I}$ & DATA \\
\hline 1040_1_6c_H1_V3 & A & NAME \\
\hline 1040_1_6c_H2_V3 & ICON & DATA \\
\hline 1040_1_6c_H3_V3 & A & SSN \\
\hline 1040_1_6c_H4_V3 & A & DATA \\
\hline 1040_1_6c_H5_V3 & $\mathrm{I}$ & DATA \\
\hline 1040_1_6c_H6_V3 & $\mathrm{I}$ & DATA \\
\hline 1040_1_6c_H1_V4 & A & NAME \\
\hline 1040_1_6c_H2_V4 & ICON & DATA \\
\hline 1040_1_6c_H3_V4 & A & SSN \\
\hline 1040_1_6c_H4_V4 & A & DATA \\
\hline 1040_1_6c_H5_V4 & I & DATA \\
\hline 1040_1_6c_H1_V5 & A & NAME \\
\hline 1040_1_6c_H2_V5 & ICON & DATA \\
\hline 1040_1_6c_H3_V5 & A & SSN \\
\hline 1040_1_6c_H4_V5 & A & DATA \\
\hline 1040_1_6c_H5_V5 & I & DATA \\
\hline 1040_1_6c_H1_V6 & A & NAME \\
\hline 1040_1_6c_H2_V6 & ICON & DATA \\
\hline 1040_1_6c_H3_V6 & A & SSN \\
\hline 1040_1_6c_H4_V6 & A & DATA \\
\hline 1040_1_6c_H5_V6 & I & DATA \\
\hline 1040_1_6d & ICON & DATA \\
\hline 1040_1_6e & I & DATA \\
\hline 1040_1_7 & $\mathrm{F}$ & DATA \\
\hline 1040_1_8a & $\mathrm{F}$ & DATA \\
\hline 1040_1_8b & $\mathrm{F}$ & DATA \\
\hline 1040_1_9 & $\mathrm{F}$ & DATA \\
\hline 1040_1_10 & $\mathrm{F}$ & DATA \\
\hline 1040_1_11 & $\mathrm{F}$ & DATA \\
\hline 1040_1_12 & $\mathrm{F}$ & DATA \\
\hline 1040_1_13 & $\mathrm{F}$ & DATA \\
\hline 1040_1_14 & $\mathrm{F}$ & DATA \\
\hline 1040_1_15 & $\mathrm{F}$ & DATA \\
\hline 1040_1_16a & $\mathrm{F}$ & DATA \\
\hline 1040_1_16b & $\mathrm{F}$ & DATA \\
\hline 1040_1_17a & $\mathrm{F}$ & DATA \\
\hline 1040_1_17b & $\mathrm{F}$ & DATA \\
\hline 1040_1_18 & $\mathrm{F}$ & DATA \\
\hline
\end{tabular}




\begin{tabular}{|c|c|c|}
\hline \multicolumn{3}{|c|}{ FORM_1040_1_Table_A } \\
\hline Entry_Field & Type & Context \\
\hline 1040_1_19 & $\mathrm{F}$ & DATA \\
\hline 1040_1_20 & $\mathrm{F}$ & DATA \\
\hline 1040_1_21a & $\mathrm{F}$ & DATA \\
\hline 1040_1_21b & $\mathrm{F}$ & DATA \\
\hline 1040_1_22_H1 & $A$ & DATA \\
\hline 1040_1_22_H2 & $\mathrm{F}$ & DATA \\
\hline 1040_1_23 & $\mathrm{F}$ & DATA \\
\hline 1040_1_24 & $\mathrm{F}$ & DATA \\
\hline 1040_1_25a & $\mathrm{F}$ & DATA \\
\hline 1040_1_25b & $\mathrm{F}$ & DATA \\
\hline 1040_1_26 & $F$ & DATA \\
\hline 1040_1_27 & $\mathrm{F}$ & DATA \\
\hline 1040_1_28 & $\mathrm{F}$ & DATA \\
\hline 1040_1_29_V1 & $A$ & NAME \\
\hline 1040_1_29_H1_V2 & A & SSN \\
\hline 1040_1_29_H2_V2 & $\mathrm{F}$ & DATA \\
\hline
\end{tabular}




\section{Form 10402 - Table A}

\begin{tabular}{|c|c|c|}
\hline \multicolumn{3}{|c|}{ FORM_1040_2_Table_A } \\
\hline Entry_Field & Type & Context \\
\hline $1040 \_232$ & $F$ & DATA \\
\hline 1040_2_33a_H1 & ICON & DATA \\
\hline 1040_2_33a_H2 & ICON & DATA \\
\hline 1040_2_33a_H3 & ICON & DATA \\
\hline 1040_2_33a_H4 & ICON & DATA \\
\hline 1040_2_33a_H5 & 1 & DATA \\
\hline 1040_2_33b & ICON & DATA \\
\hline $1040 \_2 \_33 c$ & ICON & DATA \\
\hline 1040_2_34_H1 & ICON & DATA \\
\hline $10402234 \mathrm{H} 2$ & $\mathrm{~F}$ & DATA \\
\hline $1040 \_2 \_35$ & $F$ & DATA \\
\hline $1040 \_2 \_36$ & $\mathrm{~F}$ & DATA \\
\hline 1040_2_37_V1 & $\mathrm{F}$ & DATA \\
\hline 1040_2_37_V2 & ICON & DATA \\
\hline 1040_2_38_H1 & ICON & DATA \\
\hline 1040_2_38_H2 & ICON & DATA \\
\hline $1040238 \mathrm{H} 3$ & ICON & DATA \\
\hline $1040 \_2 \_38 \mathrm{H} 4$ & $F$ & DATA \\
\hline 1040_2_39_H1 & ICON & DATA \\
\hline 1040_2_39_H2 & ICON & DATA \\
\hline 1040_2_39_H3 & $\mathrm{F}$ & DATA \\
\hline 1040_2_40 & $F$ & DATA \\
\hline $1040 \_241$ & $\mathrm{~F}$ & DATA \\
\hline 1040_2_42 & $\mathrm{F}$ & DATA \\
\hline $1040 \_2 \_43$ & $F$ & DATA \\
\hline 1040_2_44_H1 & ICON & DATA \\
\hline 1040_2_44_H2 & ICON & DATA \\
\hline 1040_2_44_H3 & A & DATA \\
\hline 1040_2_44_H4 & $F$ & DATA \\
\hline 1040_2_45 & $\mathrm{F}$ & DATA \\
\hline $1040 \_2 \_46$ & $\mathrm{~F}$ & DATA \\
\hline 1040_2_47 & $\mathrm{F}$ & DATA \\
\hline $1040 \_48$ & $F$ & DATA \\
\hline $1040 \_2449$ & $\mathrm{~F}$ & DATA \\
\hline 1040_2_50_H1 & ICON & DATA \\
\hline
\end{tabular}




\begin{tabular}{|c|c|c|}
\hline \multicolumn{3}{|c|}{ FORM_1040_2_Table_A } \\
\hline Entry_Field & Type & Context \\
\hline $1040 \_$2_50_H2 & ICON & DATA \\
\hline $1040250 \mathrm{H} 3$ & $\mathrm{~F}$ & DATA \\
\hline $1040 \_2 \_52$ & $F$ & DATA \\
\hline $1040 \_2 \_53$ & $\mathrm{~F}$ & DATA \\
\hline 1040_2_54_H1 & ICON & DATA \\
\hline 1040_2_54_H2 & $\mathrm{F}$ & DATA \\
\hline $1040 \_2 \_55$ & $F$ & DATA \\
\hline $1040 \_2 \_56$ & $\mathrm{~F}$ & DATA \\
\hline 1040257 & $\mathrm{~F}$ & DATA \\
\hline $1040 \_58$ & $\mathrm{~F}$ & DATA \\
\hline $1040 \_2 \_59$ & $F$ & DATA \\
\hline $1040 \_2 \_60$ & $\mathrm{~F}$ & DATA \\
\hline $1040 \_2 \_61$ & $F$ & DATA \\
\hline 10402262 & $F$ & DATA \\
\hline $1040 \_2 \_63$ & $F$ & DATA \\
\hline $1040 \_2 \_64$ & $\mathrm{~F}$ & DATA \\
\hline $1040265 \mathrm{~V} 1$ & $\mathrm{~F}$ & DATA \\
\hline 1040_2_65_H1_V2 & ICON & DATA \\
\hline 1040_2_65_H2_V2 & $\mathrm{F}$ & DATA \\
\hline 1040_2_S_H1_V1 & ICON & DATA \\
\hline 1040_2_S_H2_V1 & $A$ & DATA \\
\hline 1040_2_S_H3_V1 & A & DATA \\
\hline 1040_2_S_H1_V2 & ICON & DATA \\
\hline 1040_2_S_H2_V2 & A & DATA \\
\hline 1040_2_S_H3_V2 & A & DATA \\
\hline 1040_2_S_H1_V3 & ICON & DATA \\
\hline 1040_2_S_H2_V3 & A & DATA \\
\hline 1040_2_S_H3_V3 & ICON & DATA \\
\hline 1040_2_S_H4_V3 & $A$ & SSN \\
\hline 1040_2_S_H1_V4 & $A$ & DATA \\
\hline 1040_2_S_H2_V4 & A & DATA \\
\hline 1040_2_S_H1_V5 & A & DATA \\
\hline 1040_2_S_H2_V5 & $A$ & DATA \\
\hline
\end{tabular}




\section{Form 21061 - Table A}

\begin{tabular}{|c|c|c|}
\hline \multicolumn{3}{|c|}{ Form 21061 - Table A } \\
\hline Entry Field & Type & Context \\
\hline 2106_1_L_H1 & A & NAME \\
\hline 2106_1_L_H2 & A & SSN \\
\hline 2106_1_L_H3 & $A$ & DATA \\
\hline 210611 & $F$ & DATA \\
\hline 210612 & $F$ & DATA \\
\hline $2106 \quad 13$ & $\mathrm{~F}$ & DATA \\
\hline $2106 \_1 \_4$ & $\mathrm{~F}$ & DATA \\
\hline 2106_1_5 & $\mathrm{F}$ & DATA \\
\hline 2106_1_6_H1 & $\mathrm{F}$ & DATA \\
\hline 2106_1_6_H2 & $\mathrm{F}$ & DATA \\
\hline 2106_1_7_H1 & $\mathrm{F}$ & DATA \\
\hline 2106_1_7_H2 & $\mathrm{F}$ & DATA \\
\hline 2106_1_8_H1 & $\mathrm{F}$ & DATA \\
\hline 2106_1_8_H2 & $\mathrm{F}$ & DATA \\
\hline 2106_1_9 & $\mathrm{F}$ & DATA \\
\hline 2106_1_10_H1 & $\mathrm{F}$ & DATA \\
\hline 2106_1_10_H2 & $\mathrm{F}$ & DATA \\
\hline 2106_1_11_H1 & $\mathrm{F}$ & DATA \\
\hline 2106_1_11_H2 & $\mathrm{F}$ & DATA \\
\hline 2106_1_12_H1 & $\mathrm{F}$ & DATA \\
\hline 2106_1_12_H2 & $\mathrm{F}$ & DATA \\
\hline 2106_1_13 & $\mathrm{F}$ & DATA \\
\hline 2106_1_14_H1 & $\mathrm{F}$ & DATA \\
\hline 2106_1_14_H2 & $\mathrm{F}$ & DATA \\
\hline 2106_1_15 & $\mathrm{F}$ & DATA \\
\hline 2106_1_16_H1 & $\mathrm{F}$ & DATA \\
\hline 2106_1_16_H2 & $\mathrm{F}$ & DATA \\
\hline 2106_1_17 & $\mathrm{F}$ & DATA \\
\hline
\end{tabular}


Form 21062 - Table A

\begin{tabular}{|c|c|c|}
\hline \multicolumn{3}{|c|}{ Form 21062 - Table A } \\
\hline Entry Field & Type & Context \\
\hline 2106_2_A_1_H1 & A & DATA \\
\hline 2106_2_A_1_H2 & A & DATA \\
\hline 2106_2_A_2_H1 & $\mathrm{I}$ & DATA \\
\hline 2106_2_A_2_H2 & $\mathrm{I}$ & DATA \\
\hline 2106_2_A_3_H1 & 1 & DATA \\
\hline 2106_2_A_3_H2 & $\mathrm{I}$ & DATA \\
\hline 2106_2_A_4_H1 & FPER & DATA \\
\hline $2106 \_2 \_\mathrm{A} \_4 \_\mathrm{H} 2$ & FPER & DATA \\
\hline 2106_2_A_5_H1 & I & DATA \\
\hline 2106_2_A_5_H2 & 1 & DATA \\
\hline 2106_2_A_6_H1 & $\mathrm{I}$ & DATA \\
\hline 2106_2_A_6_H2 & 1 & DATA \\
\hline 2106_2_A_7_H1 & I & DATA \\
\hline 2106_2_A_7_H2 & 1 & DATA \\
\hline 2106_2_A_8_H1 & ICON & DATA \\
\hline 2106_2_A_8_H2 & ICON & DATA \\
\hline 2106_2_A_9_H1 & ICON & DATA \\
\hline 2106_2_A_9_H2 & ICON & DATA \\
\hline 2106_2_A_9_H3 & ICON & DATA \\
\hline 2106_2_A_10_H1 & ICON & DATA \\
\hline 2106_2_A_10_H2 & ICON & DATA \\
\hline 2106_2_A_10_H3 & ICON & DATA \\
\hline 2106_2_A_10_H4 & ICON & DATA \\
\hline 2106_2_B_11 & 1 & DATA \\
\hline 2106_2_B_12 & $\mathrm{I}$ & DATA \\
\hline $2106 \_2$ B_13 & $\mathrm{F}$ & DATA \\
\hline 2106_2_B_14 & $\mathrm{F}$ & DATA \\
\hline 2106_2_B_15 & $\mathrm{F}$ & DATA \\
\hline 2106_2_C_16_H1 & $\mathrm{F}$ & DATA \\
\hline 2106_2_C_16_H2 & $\mathrm{F}$ & DATA \\
\hline 2106_2_C_17_H1 & $\mathrm{F}$ & DATA \\
\hline 2106_2_C_17_H2 & $\mathrm{F}$ & DATA \\
\hline 2106_2_C_18_H1 & $\mathrm{F}$ & DATA \\
\hline 2106_2_C_18_H2 & $\mathrm{F}$ & DATA \\
\hline
\end{tabular}




\begin{tabular}{|l|l|r|}
\hline \multicolumn{3}{|c|}{ Form 2106 2 - Table A } \\
\hline Entry Field & Type & Context \\
\hline 2106_2_C_19_H1 & F & DATA \\
\hline 2106_2_C_19_H2 & F & DATA \\
\hline 2106_2_C_20_H1 & F & DATA \\
\hline 2106_2_C_20_H2 & F & DATA \\
\hline 2106_2_C_21_H1 & F & DATA \\
\hline 2106_2_C_21_H2 & F & DATA \\
\hline 2106_2_C_22_H1 & F & DATA \\
\hline 2106_2_C_22_H2 & F & DATA \\
\hline 2106_2_D_a_V1 & FP & DATA \\
\hline 2106_2_D_b_V1 & FP & DATA \\
\hline 2106_2_D_c_V1 & A & DATA \\
\hline 2106_2_D_d_V1 & FP & DATA \\
\hline 2106_2_D_e_V1 & FP & DATA \\
\hline 2106_2_D_f_V1 & FP & DATA \\
\hline 2106_2_D_a_V2 & FP & DATA \\
\hline 2106_2_D_b_V2 & FP & DATA \\
\hline 2106_2_D_c_V2 & A & DATA \\
\hline 2106_2_D_d_V2 & FP & DATA \\
\hline 2106_2_D_e_V2 & FP & DATA \\
\hline 2106_2_D_f_V2 & FP & DATA \\
\hline
\end{tabular}


Form 2441 - Table A

\begin{tabular}{|c|c|c|}
\hline \multicolumn{3}{|c|}{ Form 2441 - Table A } \\
\hline Entry Field & Type & Context \\
\hline 2441_L_H2 & $A$ & SSN \\
\hline 2441_1 & I & DATA \\
\hline 2441_2 & $\mathrm{F}$ & DATA \\
\hline 2441_3a & $F$ & DATA \\
\hline 2441_3b & $\mathrm{F}$ & DATA \\
\hline 2441_3c & $\mathrm{F}$ & DATA \\
\hline 2441_4 & $\mathrm{F}$ & DATA \\
\hline 2441_5 & $\mathrm{FF}$ & DATA \\
\hline 2441_6 & $\mathrm{F}$ & DATA \\
\hline 2441_7 & $F$ & DATA \\
\hline 2441_8 & $\mathrm{F}$ & DATA \\
\hline
\end{tabular}


Form 45621 - Table A

\begin{tabular}{|c|c|c|}
\hline \multicolumn{3}{|c|}{ Form 45621 - Table_A } \\
\hline Entry Field & Type & Context \\
\hline 4562_1_L_H1_V1 & A & NAME \\
\hline 4562_1_L_H2_V1 & A & SSN \\
\hline 4562_1_L_V2 & $A$ & DATA \\
\hline 4562_1_I_1_a_V1 & $A$ & DATA \\
\hline 4562_1_I_1_b_V1 & A & DATA \\
\hline 4562_1_I_1_c_V1 & FP & DATA \\
\hline 4562_1_I_1_d_V1 & FP & DATA \\
\hline 4562_1_I_1_a_V2 & A & DATA \\
\hline 4562_1_I_1_b_V2 & A & DATA \\
\hline 4562_1_I_1_c_V2 & FP & DATA \\
\hline 4562_1_I_1_d_V2 & FP & DATA \\
\hline 4562_1_I_2 & $\mathrm{FP}$ & DATA \\
\hline 4562_1_I_3 & FP & DATA \\
\hline 4562_1_1_4 & $\mathrm{FP}$ & DATA \\
\hline 4562_1_1_5 & $\mathrm{FP}$ & DATA \\
\hline 4562_1_I_6a_c & FP & DATA \\
\hline 4562 1_l6a_d & 1 & DATA \\
\hline 4562_1_I_6a_e & A & DATA \\
\hline 4562_1_I_6a_f & $\mathrm{FP}$ & DATA \\
\hline 4562_1_I_6b_c & $\mathrm{FP}$ & DATA \\
\hline 4562_1_l6b_d & 1 & DATA \\
\hline 4562_1_l6b_e & A & DATA \\
\hline 4562 1_I_6b_f & FP & DATA \\
\hline 4562 1_I $6 \mathrm{c}$ c $\mathrm{c}$ & FP & DATA \\
\hline 4562_1_I_6c_d & I & DATA \\
\hline 4562_1_l_6c_e & A & DATA \\
\hline 4562_1_I_6c_f & $\mathrm{FP}$ & DATA \\
\hline 4562_1_I_6d_c & FP & DATA \\
\hline 4562_1_I_6d_d & I & DATA \\
\hline 4562_1_I_6d_e & A & DATA \\
\hline 4562_1_I_6d_f & $\mathrm{FP}$ & DATA \\
\hline 4562_1_I_6e_c & FP & DATA \\
\hline 4562_1_I_6e_d & 1 & DATA \\
\hline
\end{tabular}




\begin{tabular}{|c|c|c|}
\hline \multicolumn{3}{|c|}{ Form 45621 - Table_A } \\
\hline Entry Field & Type & Context \\
\hline 4562_1_6e_e & A & DATA \\
\hline 4562_1_I_6e f & FP & DATA \\
\hline 4562_1_I_6f_c & FP & DATA \\
\hline 4562_1_I_6f_d & $\mathrm{I}$ & DATA \\
\hline 4562_1_I_6f_e & A & DATA \\
\hline 4562 1_I_6f_f & FP & DATA \\
\hline 4562_1_l6g_b_V1 & A & DATA \\
\hline 4562_1_I_6g_c_V1 & FP & DATA \\
\hline 4562 1_l $6 g$ d_V1 & I & DATA \\
\hline 4562 1_l $6 g$ e $V 1$ & $A$ & DATA \\
\hline $45621 \mathrm{I} 6 \mathrm{~g} f \mathrm{~V} 1$ & FP & DATA \\
\hline 4562_1_l_6g_b_V2 & $A$ & DATA \\
\hline 4562 1_6g_c V2 & FP & DATA \\
\hline 4562 1_l6g_d_V2 & I & DATA \\
\hline 4562_1_l_6g_e_V2 & A & DATA \\
\hline 4562_1_I_6g_f_V2 & FP & DATA \\
\hline 4562_1_I_6h_b_V1 & A & DATA \\
\hline 4562_1_I_6h_c_V1 & FP & DATA \\
\hline 4562_1_l6h_d_V1 & $\mathrm{I}$ & DATA \\
\hline 4562_1_l_6h_e_v1 & A & DATA \\
\hline 4562 1_I_6h_f_v1 & FP & DATA \\
\hline 4562_1_I_6h_b_v2 & A & DATA \\
\hline 4562_1_l6h_c_V2 & FP & DATA \\
\hline 4562_1_l_6h_d_V2 & I & DATA \\
\hline 4562_1_ $6 h \_e \_v 2$ & A & DATA \\
\hline 4562_1_6hffv2 & FP & DATA \\
\hline 4562_1_I_7_b & A & DATA \\
\hline $4562117 \mathrm{c}$ & FP & DATA \\
\hline 4562 1_ I d d & I & DATA \\
\hline $45621 \mathrm{I} e$ & A & DATA \\
\hline 4562_1_I_7ff & FP & DATA \\
\hline 4562 1_18 & FP & DATA \\
\hline 4562 1_1_9 & FP & DATA \\
\hline 4562_1_I_10 & FP & DATA \\
\hline 4562_1_I_11 & FP & DATA \\
\hline 4562_1_I_12 & FP & DATA \\
\hline $45621 \mid 13$ & FP & DATA \\
\hline
\end{tabular}




\begin{tabular}{|l|c|c|}
\hline \multicolumn{3}{|c|}{ Form 4562 1 - Table_A } \\
\hline Entry Field & Type & Context \\
\hline 4562_1_II_1_a_V1 & A & DATA \\
\hline 4562_1_II_1_b_V1 & A & DATA \\
\hline 4562_1_II_1_c_V1 & FP & DATA \\
\hline 4562_1_II_1_d_V1 & A & DATA \\
\hline 4562_1_II_1_e_V1 & I & DATA \\
\hline 4562_1_II_1_f_V1 & FP & DATA \\
\hline 4562_1_II_1_a_V2 & A & DATA \\
\hline 4562_1_II_1_b_V2 & A & DATA \\
\hline 4562_1_II_1_c_V2 & FP & DATA \\
\hline 4562_1_II_1_d_V2 & A & DATA \\
\hline 4562_1_II_1_e_V2 & I & DATA \\
\hline 4562_1_II_1_f_V2 & FP & DATA \\
\hline 4562_1_II_2 & FP & DATA \\
\hline 4562_1_II_3 & FP & DATA \\
\hline
\end{tabular}




\section{Form 45622 - Table}

\begin{tabular}{|c|c|c|}
\hline \multicolumn{3}{|c|}{ Form 45622 - Table A } \\
\hline Entry Field & Type & Context \\
\hline 4562_2_A_H1 & ICON & DATA \\
\hline 4562_2_A_H2 & ICON & DATA \\
\hline 4562_2_A_H3 & ICON & DATA \\
\hline 4562_2_A_H4 & ICON & DATA \\
\hline 4562_2_A_a_V1 & $A$ & DATA \\
\hline 4562_2_A_b_V1 & $A$ & DATA \\
\hline 4562_2_A_C_V1 & I & DATA \\
\hline 4562_2_A_d_V1 & FP & DATA \\
\hline 4562_2_A_e_V1 & $\mathrm{FP}$ & DATA \\
\hline 4562_2_A_f_V1 & $A$ & DATA \\
\hline 4562_2_A_g_V1 & FP & DATA \\
\hline 4562_2_A_h_V1 & FP & DATA \\
\hline 4562_2_A_a_V2 & A & DATA \\
\hline 4562_2_A_b_V2 & A & DATA \\
\hline 4562_2_A_C_V2 & I & DATA \\
\hline 4562_2_A_d_V2 & FP & DATA \\
\hline 4562_2_A_e_V2 & FP & DATA \\
\hline 4562_2_A_f_V2 & A & DATA \\
\hline 4562_2_A_g_V2 & $\mathrm{FP}$ & DATA \\
\hline 4562_2_A_h_V2 & FP & DATA \\
\hline 4562_2_A_a_V3 & A & DATA \\
\hline 4562_2_A_b_V3 & A & DATA \\
\hline 4562_2_A_C_V3 & 1 & DATA \\
\hline 4562_2_A_d_V3 & $\mathrm{FP}$ & DATA \\
\hline 4562_2_A_e_V3 & $\mathrm{FP}$ & DATA \\
\hline 4562_2_A_f_V3 & A & DATA \\
\hline 4562_2_A_g_V3 & $\mathrm{FP}$ & DATA \\
\hline 4562_2_A_h_V3 & $\mathrm{FP}$ & DATA \\
\hline 4562_2_A_a_V4 & $A$ & DATA \\
\hline 4562_2_A_b_V4 & $A$ & DATA \\
\hline 4562_2_A_C_V4 & 1 & DATA \\
\hline 4562_2_A_d_V4 & $\mathrm{FP}$ & DATA \\
\hline 4562_2_A_e_V4 & $\mathrm{FP}$ & DATA \\
\hline 4562_2_A_f_V4 & A & DATA \\
\hline
\end{tabular}




\begin{tabular}{|c|c|c|}
\hline \multicolumn{3}{|c|}{ Form 45622 - Table A } \\
\hline Entry Field & Type & Context \\
\hline 4562_2_A_g_V4 & $\mathrm{FP}$ & DATA \\
\hline 4562_2_A_h_V4 & $\mathrm{FP}$ & DATA \\
\hline 4562_2_A_a_V5 & A & DATA \\
\hline 4562_2_A_b_V5 & A & DATA \\
\hline 4562_2_A_C_V5 & 1 & DATA \\
\hline 4562_2_A_d_V5 & FP & DATA \\
\hline 4562_2_A_e_V5 & FP & DATA \\
\hline 4562_2_A_f_V5 & A & DATA \\
\hline 4562_2_A_g_V5 & $\mathrm{FP}$ & DATA \\
\hline 4562_2_A_h_V5 & $\mathrm{FP}$ & DATA \\
\hline 4562_2_A_a_V6 & A & DATA \\
\hline 4562_2_A_b_V6 & A & DATA \\
\hline 4562_2_A_C_V6 & I & DATA \\
\hline 4562_2_A_d_V6 & $\mathrm{FP}$ & DATA \\
\hline 4562_2_A_e_V6 & FP & DATA \\
\hline 4562_2_A_f_V6 & A & DATA \\
\hline 4562_2_A_g_V6 & FP & DATA \\
\hline 4562_2_A_h_V6 & $\mathrm{FP}$ & DATA \\
\hline 4562_2_A_a_V7 & A & DATA \\
\hline 4562_2_A_b_V7 & A & DATA \\
\hline 4562_2_A_C_V7 & 1 & DATA \\
\hline 4562_2_A_d_V7 & $\mathrm{FP}$ & DATA \\
\hline 4562_2_A_e_V7 & $\mathrm{FP}$ & DATA \\
\hline 4562_2_A_f_V7 & A & DATA \\
\hline 4562_2_A_g_V7 & FP & DATA \\
\hline 4562_2_A_h_V7 & FP & DATA \\
\hline 4562_2_A_a_V8 & A & DATA \\
\hline 4562_2_A_b_V8 & A & DATA \\
\hline 4562_2_A_C_V8 & 1 & DATA \\
\hline 4562_2_A_d_V8 & $\mathrm{FP}$ & DATA \\
\hline 4562_2_A_e_V8 & $\mathrm{FP}$ & DATA \\
\hline 4562_2_A_f_V8 & A & DATA \\
\hline 4562_2_A_g_V8 & $\mathrm{FP}$ & DATA \\
\hline 4562_2_A_h_V8 & $\mathrm{FP}$ & DATA \\
\hline 4562_2_A_a_V9 & A & DATA \\
\hline 4562_2_A_b_V9 & A & DATA \\
\hline 4562_2_A_C_V9 & 1 & DATA \\
\hline
\end{tabular}




\begin{tabular}{|c|c|c|}
\hline \multicolumn{3}{|c|}{ Form 45622 - Table A } \\
\hline Entry Field & Type & Context \\
\hline 4562_2_A_d_V9 & $\mathrm{FP}$ & DATA \\
\hline 4562_2_A_e_V9 & $\mathrm{FP}$ & DATA \\
\hline 4562_2_A_f_V9 & A & DATA \\
\hline 4562_2_A_g_V9 & $\mathrm{FP}$ & DATA \\
\hline 4562_2_A_h_V9 & $\mathrm{FP}$ & DATA \\
\hline 4562_2_A_h_V10 & $\mathrm{FP}$ & DATA \\
\hline 4562_2_A_g_V11 & $\mathrm{FP}$ & DATA \\
\hline 4562_2_B_1_1 & 1 & DATA \\
\hline 4562_2_B_1_2 & I & DATA \\
\hline 4562_2_B_1_3 & I & DATA \\
\hline 4562_2_B_1_4 & 1 & DATA \\
\hline 4562_2_B_1_5 & 1 & DATA \\
\hline 4562_2_B_1_6 & I & DATA \\
\hline 4562_2_B_2_1 & 1 & DATA \\
\hline 4562_2_B_2_2 & 1 & DATA \\
\hline 4562_2_B_2_3 & 1 & DATA \\
\hline 4562_2_B_2_4 & 1 & DATA \\
\hline 4562_2_B_2_5 & 1 & DATA \\
\hline 4562_2_B_2_6 & 1 & DATA \\
\hline 4562_2_B_3_1 & 1 & DATA \\
\hline 4562_2_B_3_2 & 1 & DATA \\
\hline 4562_2_B_3_3 & 1 & DATA \\
\hline 4562_2_B_3_4 & 1 & DATA \\
\hline 4562_2_B_3_5 & 1 & DATA \\
\hline 4562_2_B_3_6 & 1 & DATA \\
\hline 4562_2_B_4_1 & 1 & DATA \\
\hline 4562_2_B_4_2 & 1 & DATA \\
\hline 4562_2_B_4_3 & I & DATA \\
\hline 4562_2_B_4_4 & I & DATA \\
\hline 4562_2_B_4_5 & 1 & DATA \\
\hline 4562_2_B_4_6 & 1 & DATA \\
\hline 4562_2_B_5_1_H1 & ICON & DATA \\
\hline 4562_2_B_5_1_H2 & ICON & DATA \\
\hline 4562_2_B_5_2_H1 & ICON & DATA \\
\hline 4562_2_B_5_2_H2 & ICON & DATA \\
\hline 4562_2_B_5_3_H1 & ICON & DATA \\
\hline 4562_2_B_5_3_H2 & ICON & DATA \\
\hline
\end{tabular}




\begin{tabular}{|c|c|c|}
\hline \multicolumn{3}{|c|}{ Form 45622 - Table A } \\
\hline Entry Field & Type & Context \\
\hline 4562_2_B_5_4_H1 & ICON & DATA \\
\hline 4562_2_B_5_4_H2 & ICON & DATA \\
\hline 4562_2_B_5_5_H1 & ICON & DATA \\
\hline 4562_2_B_5_5_H2 & ICON & DATA \\
\hline 4562_2_B_5_6_H1 & ICON & DATA \\
\hline 4562_2_B_5_6_H2 & ICON & DATA \\
\hline 4562_2_B_6_1_H1 & ICON & DATA \\
\hline 4562_2_B_6_1_H2 & ICON & DATA \\
\hline 4562_2_B_6_2_H1 & ICON & DATA \\
\hline 4562_2_B_6_2_H2 & ICON & DATA \\
\hline 4562_2_B_6_3_H1 & ICON & DATA \\
\hline 4562_2_B_6_3_H2 & ICON & DATA \\
\hline 4562_2_B_6_4_H1 & ICON & DATA \\
\hline 4562_2_B_6_4_H2 & ICON & DATA \\
\hline 4562_2_B_6_5_H1 & ICON & DATA \\
\hline 4562_2_B_6_5_H2 & ICON & DATA \\
\hline 4562_2_B_6_6_H1 & ICON & DATA \\
\hline 4562_2_B_6_6_H2 & ICON & DATA \\
\hline 4562_2_B_7_1_H1 & ICON & DATA \\
\hline 4562_2_B_7_1_H2 & ICON & DATA \\
\hline 4562_2_B_7_2_H1 & ICON & DATA \\
\hline 4562_2_B_7_2_H2 & ICON & DATA \\
\hline 4562_2_B_7_3_H1 & ICON & DATA \\
\hline 4562_2_B_7_3_H2 & ICON & DATA \\
\hline 4562_2_B_7_4_H1 & ICON & DATA \\
\hline 4562_2_B_7_4_H2 & ICON & DATA \\
\hline 4562_2_B_7_5_H1 & ICON & DATA \\
\hline 4562_2_B_7_5_H2 & ICON & DATA \\
\hline 4562_2_B_7_6_H1 & ICON & DATA \\
\hline 4562_2_B_7_6_H2 & ICON & DATA \\
\hline 4562_2_C_8_H1 & ICON & DATA \\
\hline 4562_2_C_8_H2 & ICON & DATA \\
\hline 4562_2_C_9_H1 & ICON & DATA \\
\hline 4562_2_C_9_H2 & ICON & DATA \\
\hline 4562_2_C_10_H1 & ICON & DATA \\
\hline 4562_2_C_10_H2 & ICON & DATA \\
\hline 4562_2_C_11_H1 & ICON & DATA \\
\hline
\end{tabular}




\begin{tabular}{|l|c|c|}
\hline \multicolumn{3}{|c|}{ Form 4562 2 - Table A } \\
\hline Entry Field & Type & Context \\
\hline 4562_2_C_11_H2 & ICON & DATA \\
\hline 4562_2_C_12_H1 & ICON & DATA \\
\hline 4562_2_C_12_H2 & ICON & DATA \\
\hline
\end{tabular}


Form 6251 - Table A

\begin{tabular}{|c|c|c|}
\hline \multicolumn{3}{|c|}{ Form 6251 - Table A } \\
\hline Entry Field & Type & Context \\
\hline 6251_L_H1 & A & NAME \\
\hline 6251_L_H2 & $A$ & SSN \\
\hline 6251_1 & $\mathrm{F}$ & DATA \\
\hline 6251_2 & $\mathrm{F}$ & DATA \\
\hline 6251_3 & $\mathrm{F}$ & DATA \\
\hline $6251 \_4 a$ & $\mathrm{~F}$ & DATA \\
\hline 6251_4b & $\mathrm{F}$ & DATA \\
\hline 6251_4c & $\mathrm{F}$ & DATA \\
\hline 6251_4d & $\mathrm{F}$ & DATA \\
\hline 6251_4e & $\mathrm{F}$ & DATA \\
\hline $6251 \_4 f$ & $\mathrm{FU}$ & DATA \\
\hline 6251_4g & $\mathrm{F}$ & DATA \\
\hline $6251 \_4 h$ & $\mathrm{~F}$ & DATA \\
\hline $6251 \_4 i$ & $\mathrm{~F}$ & DATA \\
\hline $6251 \_4 j$ & $\mathrm{~F}$ & DATA \\
\hline 6251_4k & $\mathrm{F}$ & DATA \\
\hline 6251 4I & $\mathrm{F}$ & DATA \\
\hline $6251 \_4 m$ & $\mathrm{~F}$ & DATA \\
\hline $6251 \_4 n$ & $\mathrm{~F}$ & DATA \\
\hline $6251 \_40$ & $\mathrm{~F}$ & DATA \\
\hline $6251 \_4 p$ & $\mathrm{~F}$ & DATA \\
\hline $6251 \_4 q$ & $\mathrm{~F}$ & DATA \\
\hline $6251 \_4 r$ & $\mathrm{~F}$ & DATA \\
\hline $6251 \_4 s$ & $\mathrm{~F}$ & DATA \\
\hline $6251 \_4 \mathrm{t}$ & $\mathrm{F}$ & DATA \\
\hline $6251 \_4 u$ & $\mathrm{~F}$ & DATA \\
\hline 6251_5a & $F$ & DATA \\
\hline 6251_5b & $\mathrm{F}$ & DATA \\
\hline $6251 \_5 c$ & $\mathrm{~F}$ & DATA \\
\hline $6251 \_5 d$ & $F$ & DATA \\
\hline $6251 \_5 e$ & $F$ & DATA \\
\hline $6251 \_5 f$ & $F$ & DATA \\
\hline $6251 \_5 \mathrm{~g}$ & $\mathrm{~F}$ & DATA \\
\hline $6251 \_5 i$ & $F$ & DATA \\
\hline
\end{tabular}




\begin{tabular}{|c|c|c|}
\hline \multicolumn{3}{|c|}{ Form 6251 - Table A } \\
\hline Entry Field & Type & Context \\
\hline $6251 \_5 j$ & $\mathrm{~F}$ & DATA \\
\hline $6251 \_5 k$ & $\mathrm{~F}$ & DATA \\
\hline $6251 \_6$ & $\mathrm{~F}$ & DATA \\
\hline $6251 \_7$ & $\mathrm{~F}$ & DATA \\
\hline $6251 \_8$ & $\mathrm{~F}$ & DATA \\
\hline $6251 \_9$ & $\mathrm{~F}$ & DATA \\
\hline $6251 \_10$ & $F$ & DATA \\
\hline $6251 \_11$ & $F$ & DATA \\
\hline $6251 \_12$ & $\mathrm{~F}$ & DATA \\
\hline $6251 \_13$ & $\mathrm{~F}$ & DATA \\
\hline 6251_14 & $\mathrm{F}$ & DATA \\
\hline $6251 \_15$ & $F$ & DATA \\
\hline 6251_16 & $\mathrm{F}$ & DATA \\
\hline $6251 \_17$ & $\mathrm{~F}$ & DATA \\
\hline 6251_18 & $\mathrm{F}$ & DATA \\
\hline 6251_19 & $\mathrm{F}$ & DATA \\
\hline
\end{tabular}




\section{Form schedule a - Table A}

\begin{tabular}{|c|c|c|}
\hline \multicolumn{3}{|c|}{ Form sch_a - Table A } \\
\hline Entry Field & Type & Context \\
\hline SchA_L_H1 & A & NAME \\
\hline SchA_L_H2 & A & SSN \\
\hline SchA_1a & $\mathrm{F}$ & DATA \\
\hline SchA_1b_V1 & $A$ & DATA \\
\hline SchA_1b_V2 & CA & DATA \\
\hline SchA_1b_H1_V3 & $\mathrm{CA}$ & DATA \\
\hline SchA_1b_H2_V3 & $\mathrm{F}$ & DATA \\
\hline SchA_2 & $\mathrm{F}$ & DATA \\
\hline SchA_3 & $F$ & DATA \\
\hline SchA_4 & $F$ & DATA \\
\hline SchA_5 & $F$ & DATA \\
\hline SchA_6 & $\mathrm{F}$ & DATA \\
\hline SchA_7_V1 & $A$ & DATA \\
\hline SchA_7_H1_V2 & $\mathrm{CA}$ & DATA \\
\hline SchA_7_H2_V2 & $\mathrm{F}$ & DATA \\
\hline SchA_8_ & $\mathrm{F}$ & DATA \\
\hline SchA_9a & $\mathrm{F}$ & DATA \\
\hline SchA_9b_V1 & $A$ & DATA \\
\hline SchA_9b_H1_V2 & $\mathrm{CA}$ & DATA \\
\hline SchA_9b_H2_V2 & $\mathrm{F}$ & DATA \\
\hline SchA_10 & $\mathrm{F}$ & DATA \\
\hline SchA_11 & $\mathrm{F}$ & DATA \\
\hline SchA_12a & $\mathrm{F}$ & DATA \\
\hline SchA_12b & $\mathrm{F}$ & DATA \\
\hline SchA_13 & $\mathrm{F}$ & DATA \\
\hline SchA_14_H1 & A & DATA \\
\hline SchA_14_H2 & $F$ & DATA \\
\hline SchA_15 & $F$ & DATA \\
\hline SchA_16 & $F$ & DATA \\
\hline SchA_17 & $F$ & DATA \\
\hline SchA_18 & $F$ & DATA \\
\hline SchA_19 & $\mathrm{F}$ & DATA \\
\hline SchA_20_H1 & A & DATA \\
\hline
\end{tabular}




\begin{tabular}{|l|c|c|}
\hline \multicolumn{3}{|c|}{ Form sch_a - Table A } \\
\hline \multicolumn{1}{|c|}{ Entry Field } & Type & Context \\
\hline SchA_20_H2 & F & DATA \\
\hline SchA_21_V1 & A & DATA \\
\hline SchA_21_V2 & CA & DATA \\
\hline SchA_21_H1_V3 & CA & DATA \\
\hline SchA_21_H2_V3 & F & DATA \\
\hline SchA_22 & F & DATA \\
\hline SchA_23 & F & DATA \\
\hline SchA_24 & F & DATA \\
\hline SchA_25_V1 & A & DATA \\
\hline SchA_25_V2 & CA & DATA \\
\hline SchA_25_H1_V3 & CA & DATA \\
\hline SchA_25_H2_V3 & F & DATA \\
\hline SchA_26 & F & DATA \\
\hline
\end{tabular}




\section{Form Schedule b - Table A}

\begin{tabular}{|c|c|c|}
\hline \multicolumn{3}{|c|}{ Form sch_b - Table A } \\
\hline Entry Field & Type & Context \\
\hline SchB_L_H1 & $A$ & NAME \\
\hline SchB_L_H2 & $A$ & SSN \\
\hline SchB_I_1_H1 & $\mathrm{A}$ & DATA \\
\hline SchB_I_1_H2 & $\mathrm{F}$ & DATA \\
\hline SchB_I_2_H1_V1 & A & DATA \\
\hline SchB_I_2_H2_V1 & $F$ & DATA \\
\hline SchB_I_2_H1_V2 & $A$ & DATA \\
\hline SchB_I_2_H2_V2 & $F$ & DATA \\
\hline SchB_I_2_H1_V3 & $A$ & DATA \\
\hline SchB_I_2_H2_V3 & $\mathrm{F}$ & DATA \\
\hline SchB_I_2_H1_V4 & $A$ & DATA \\
\hline SchB_I_2_H2_V4 & $\mathrm{F}$ & DATA \\
\hline SchB_I_2_H1_V5 & $A$ & DATA \\
\hline SchB_I_2_H2_V5 & $\mathrm{F}$ & DATA \\
\hline SchB_I_2_H1_V6 & A & DATA \\
\hline SchB_I_2_H2_V6 & $\mathrm{F}$ & DATA \\
\hline SchB_I_2_H1_V7 & A & DATA \\
\hline SchB_I_2_H2_V7 & $\mathrm{F}$ & DATA \\
\hline SchB_I_2_H1_V8 & A & DATA \\
\hline SchB_I_2_H2_V8 & $\mathrm{F}$ & DATA \\
\hline SchB_I_2_H1_V9 & $A$ & DATA \\
\hline SchB_I_2_H2_V9 & $\mathrm{F}$ & DATA \\
\hline SchB_I_2_H1_V10 & $A$ & DATA \\
\hline SchB_I_2_H2_V10 & $\mathrm{F}$ & DATA \\
\hline SchB_I_2_H1_V11 & $A$ & DATA \\
\hline SchB_I_2_H2_V11 & $\mathrm{F}$ & DATA \\
\hline SchB_I_2_H1_V12 & A & DATA \\
\hline SchB_I_2_H2_V12 & $\mathrm{F}$ & DATA \\
\hline SchB_I_2_H1_V13 & A & DATA \\
\hline SchB_I_2_H2_V13 & $\mathrm{F}$ & DATA \\
\hline SchB_I_3 & $\mathrm{F}$ & DATA \\
\hline SchB_II_4_V1 & $\mathrm{F}$ & DATA \\
\hline SchB_II_4_H1_V2 & A & DATA \\
\hline
\end{tabular}




\begin{tabular}{|c|c|c|}
\hline \multicolumn{3}{|c|}{ Form sch_b - Table A } \\
\hline Entry Field & Type & Context \\
\hline SchB_II_4_H2_V2 & $\mathrm{F}$ & DATA \\
\hline SchB_II_4_H1_V3 & A & DATA \\
\hline SchB_II_4_H2_V3 & $\mathrm{F}$ & DATA \\
\hline SchB_II_4_H1_V4 & A & DATA \\
\hline SchB_II_4_H2_V4 & $\mathrm{F}$ & DATA \\
\hline SchB_II_4_H1_V5 & A & DATA \\
\hline SchB_II_4_H2_V5 & $\mathrm{F}$ & DATA \\
\hline SchB_II_4_H1_V6 & $A$ & DATA \\
\hline SchB_II_4_H2_V6 & $\mathrm{F}$ & DATA \\
\hline SchB_II_4_H1_V7 & $A$ & DATA \\
\hline SchB_II_4_H2_V7 & $\mathrm{F}$ & DATA \\
\hline SchB_II_4_H1_V8 & $A$ & DATA \\
\hline SchB_II_4_H2_V8 & $\mathrm{F}$ & DATA \\
\hline SchB_II_4_H1_V9 & A & DATA \\
\hline SchB_II_4_H2_V9 & $\mathrm{F}$ & DATA \\
\hline SchB_II_4_H1_V10 & A & DATA \\
\hline SchB_II_4_H2_V10 & $\mathrm{F}$ & DATA \\
\hline SchB_II_4_H1_V11 & A & DATA \\
\hline SchB_II_4_H2_V11 & $\mathrm{F}$ & DATA \\
\hline SchB_II_4_H1_V12 & A & DATA \\
\hline SchB_II_4_H2_V12 & $\mathrm{F}$ & DATA \\
\hline SchB_II_4_H1_V13 & $A$ & DATA \\
\hline SchB_II_4_H2_V13 & $\mathrm{F}$ & DATA \\
\hline SchB_II_4_H1_V14 & $A$ & DATA \\
\hline SchB_II_4_H2_V14 & $\mathrm{F}$ & DATA \\
\hline SchB_II_5 & $\mathrm{F}$ & DATA \\
\hline SchB_II_6 & $\mathrm{F}$ & DATA \\
\hline SchB_II_7 & $\mathrm{F}$ & DATA \\
\hline SchB_II_8 & $\mathrm{F}$ & DATA \\
\hline SchB_II_9 & $\mathrm{F}$ & DATA \\
\hline SchB_III_10_H1_V1 & ICON & DATA \\
\hline SchB_III_10_H2_V1 & ICON & DATA \\
\hline SchB_III_10_V2 & $A$ & DATA \\
\hline SchB_III_11_H1 & ICON & DATA \\
\hline SchB_III_11_H2 & ICON & DATA \\
\hline
\end{tabular}




\section{Form Schedule c 1 - Table A}

\begin{tabular}{|l|c|c|}
\hline \multicolumn{3}{|c|}{ Form sch_c 1 - Table A } \\
\hline \multicolumn{1}{|c|}{ Entry Field } & Type & Context \\
\hline SchC_1_L_H1 & A & NAME \\
\hline SchC_1_L_H2 & A & SSN \\
\hline SchC_1_A & A & DATA \\
\hline SchC_1_B & A & DATA \\
\hline SchC_1_C & A & DATA \\
\hline SchC_1_D & A & DATA \\
\hline SchC_1_E(1) & ICON & DATA \\
\hline SchC_1_E(2) & ICON & DATA \\
\hline SchC_1_E(3) & ICON & DATA \\
\hline SchC_1_F(1) & ICON & DATA \\
\hline SchC_1_F(2) & ICON & DATA \\
\hline SchC_1_F(3)_H1 & ICON & DATA \\
\hline SchC_1_F(3)_H2 & A & DATA \\
\hline SchC_1_G_H1 & ICON & DATA \\
\hline SchC_1_G_H2 & ICON & DATA \\
\hline SchC_1_H_H1 & ICON & DATA \\
\hline SchC_1_H_H2 & ICON & DATA \\
\hline SchC_1_IH1 & ICON & DATA \\
\hline SchC_1_I_H2 & ICON & DATA \\
\hline SchC_1_J & ICON & DATA \\
\hline SchC_1_I_1a & F & DATA \\
\hline SchC_1__1b & F & DATA \\
\hline SchC_1_I_1C & F & DATA \\
\hline SchC_1__2 & F & DATA \\
\hline SchC_1__3 & F & DATA \\
\hline SchC_1_I_4 & F & DATA \\
\hline SchC_1__5 & F & DATA \\
\hline SchC_1_I_6 & F & DATA \\
\hline SchC_1_I_7 & F & DATA \\
\hline SchC_1_I_8 & F & DATA \\
\hline SchC_1_I_9 & F & DATA \\
\hline SchC_1_I_10 & F & DATA \\
\hline SchC_1_I_11 & F & DATA \\
\hline
\end{tabular}




\begin{tabular}{|c|c|c|}
\hline \multicolumn{3}{|c|}{ Form sch_c 1 - Table A } \\
\hline Entry Field & Type & Context \\
\hline SchC_1_II_12 & $\mathrm{F}$ & DATA \\
\hline SchC_1_II_13 & $F$ & DATA \\
\hline SchC_1_II_14 & $\mathrm{F}$ & DATA \\
\hline SchC_1_II_15 & $\mathrm{F}$ & DATA \\
\hline SchC_1_II_16 & $\mathrm{F}$ & DATA \\
\hline SchC_1_II_17a & $\mathrm{F}$ & DATA \\
\hline SchC_1_II_17b & $\mathrm{F}$ & DATA \\
\hline SchC_1_II_18 & $\mathrm{F}$ & DATA \\
\hline SchC_1_II_19 & $\mathrm{F}$ & DATA \\
\hline SchC_1_II_20 & $F$ & DATA \\
\hline SchC_1_II_21 & $\mathrm{F}$ & DATA \\
\hline SchC_1_II_22 & $\mathrm{F}$ & DATA \\
\hline SchC_1_II_23 & $\mathrm{F}$ & DATA \\
\hline SchC_1_II_24 & $\mathrm{F}$ & DATA \\
\hline SchC_1_II_25 & $\mathrm{F}$ & DATA \\
\hline SchC_1_II_26a & $\mathrm{F}$ & DATA \\
\hline SchC_1_II_26b & $\mathrm{F}$ & DATA \\
\hline SchC $1 \| 26 c$ & $\mathrm{~F}$ & DATA \\
\hline SchC $1 \| 26 d$ & $\mathrm{~F}$ & DATA \\
\hline SchC_1_II_27 & $\mathrm{F}$ & DATA \\
\hline SchC_1_II_28a & $\mathrm{F}$ & DATA \\
\hline SchC_1_II_28b & $\mathrm{F}$ & DATA \\
\hline SchC_1_II_28c & $\mathrm{F}$ & DATA \\
\hline SchC_1_II_29_V1 & A & DATA \\
\hline SchC_1_II_29_V2 & A & DATA \\
\hline SchC_1_II_29_V3 & A & DATA \\
\hline SchC_1_II_29_V4 & A & DATA \\
\hline SchC_1_II_29_V5 & $A$ & DATA \\
\hline SchC_1_II_29_V6 & $F$ & DATA \\
\hline SchC_1_II_30 & $\mathrm{F}$ & DATA \\
\hline SchC_1_II_31 & $\mathrm{F}$ & DATA \\
\hline SchC_1_II_32a & ICON & DATA \\
\hline SchC_1_II_32b & ICON & DATA \\
\hline
\end{tabular}




\section{Form Schedule c 2 - Table A}

\begin{tabular}{|l|c|c|}
\hline \multicolumn{3}{|c|}{ Form sch_c 2 - Table A } \\
\hline \multicolumn{1}{|c|}{ Entry Field } & Type & Context \\
\hline SchC_2_1 & F & DATA \\
\hline SchC_2_2 & F & DATA \\
\hline SchC_2_3 & F & DATA \\
\hline SchC_2_4 & F & DATA \\
\hline SchC_2_5 & F & DATA \\
\hline SchC_2_6 & F & DATA \\
\hline SchC_2_7 & F & DATA \\
\hline SchC_2_8 & F & DATA \\
\hline
\end{tabular}




\section{Form Schedule d 1- Table A}

\begin{tabular}{|c|c|c|}
\hline \multicolumn{3}{|c|}{ Form sch_d 1 - Table A } \\
\hline Entry Field & Type & Context \\
\hline SchD_1_L_H1 & $A$ & NAME \\
\hline SchD_1_L_H2 & A & SSN \\
\hline SchD_1_1 & $\mathrm{F}$ & DATA \\
\hline SchD_1_I_2a_a_V1 & $A$ & DATA \\
\hline SchD_1_I_2a_b_V1 & A & DATA \\
\hline SchD_1_I_2a_c_V1 & $A$ & DATA \\
\hline SchD_1_I_2a_d_V1 & $\mathrm{F}$ & DATA \\
\hline SchD_1_I_2a_e_V1 & $\mathrm{F}$ & DATA \\
\hline SchD_1_I_2a_f_V1 & $\mathrm{F}$ & DATA \\
\hline SchD_1_I_2a_g_V1 & $\mathrm{F}$ & DATA \\
\hline SchD_1_I_2a_a_V2 & A & DATA \\
\hline SchD_1_I_2a_b_v2 & A & DATA \\
\hline SchD_1_I_2a_c_V2 & $A$ & DATA \\
\hline SchD_1_I_2a_d_V2 & $F$ & DATA \\
\hline SchD_1_I_2a_e_V2 & $\mathrm{F}$ & DATA \\
\hline SchD_1_I_2a_f_V2 & $\mathrm{F}$ & DATA \\
\hline SchD_1_I_2a_g_v2 & $\mathrm{F}$ & DATA \\
\hline SchD_1_I_2a_a_V3 & A & DATA \\
\hline SchD_1_I_2a_b_V3 & A & DATA \\
\hline SchD_1_I_2a_c_V3 & A & DATA \\
\hline SchD_1_I_2a_d_V3 & $\mathrm{F}$ & DATA \\
\hline SchD_1_I_2a_e_V3 & $\mathrm{F}$ & DATA \\
\hline SchD_1_I_2a_f_v3 & $\mathrm{F}$ & DATA \\
\hline SchD_1_I_2a_g_V3 & $\mathrm{F}$ & DATA \\
\hline SchD_1_I_2a_a_V4 & A & DATA \\
\hline SchD_1_I_2a_b_V4 & $A$ & DATA \\
\hline SchD_1_I_2a_c_V4 & A & DATA \\
\hline SchD_1_I_2a_d_V4 & $\mathrm{F}$ & DATA \\
\hline SchD_1_I_2a_e_V4 & $\mathrm{F}$ & DATA \\
\hline SchD_1_I_2a_f_V4 & $\mathrm{F}$ & DATA \\
\hline SchD_1_I_2a_g_V4 & $F$ & DATA \\
\hline SchD_1_I_2a_a_V5 & A & DATA \\
\hline
\end{tabular}




\begin{tabular}{|c|c|c|}
\hline \multicolumn{3}{|c|}{ Form sch_d 1 - Table A } \\
\hline Entry Field & Type & Context \\
\hline SchD_1_I_2a_b_V5 & A & DATA \\
\hline SchD_1_I_2a_c_V5 & $A$ & DATA \\
\hline SchD_1_I_2a_d_V5 & $\mathrm{F}$ & DATA \\
\hline SchD_1_I_2a_e_V5 & $\mathrm{F}$ & DATA \\
\hline SchD_1_I_2a_f_V5 & $\mathrm{F}$ & DATA \\
\hline SchD_1_I_2a_g_V5 & $\mathrm{F}$ & DATA \\
\hline SchD_1_I_2a_a_V6 & A & DATA \\
\hline SchD_1_I_2a_b_V6 & A & DATA \\
\hline SchD_1_I_2a_c_V6 & A & DATA \\
\hline SchD_1_I_2a_d_V6 & $\mathrm{F}$ & DATA \\
\hline SchD_1_I_2a_e_V6 & $\mathrm{F}$ & DATA \\
\hline SchD_1_I_2a_f_V6 & $\mathrm{F}$ & DATA \\
\hline SchD_1_I_2a_g_V6 & $\mathrm{F}$ & DATA \\
\hline SchD_1_I_2a_a_V7 & A & DATA \\
\hline SchD_1_I_2a_b_V7 & A & DATA \\
\hline SchD_1_I_2a_c_V7 & A & DATA \\
\hline SchD_1_I_2a_d_v7 & $\mathrm{F}$ & DATA \\
\hline SchD_1_I_2a_e_v7 & $\mathrm{F}$ & DATA \\
\hline SchD_1_I_2a_f_v7 & $\mathrm{F}$ & DATA \\
\hline SchD_1_I_2a_g_v7 & $\mathrm{F}$ & DATA \\
\hline SchD_1_I_2a_a_v8 & A & DATA \\
\hline SchD_1_I_2a_b_V8 & A & DATA \\
\hline SchD_1_I_2a_c_V8 & A & DATA \\
\hline SchD_1_I_2a_d_V8 & $\mathrm{F}$ & DATA \\
\hline SchD_1_I_2a_e_V8 & $\mathrm{F}$ & DATA \\
\hline SchD_1_I_2a_f_v8 & $\mathrm{F}$ & DATA \\
\hline SchD_1_I_2a_g_V8 & $\mathrm{F}$ & DATA \\
\hline SchD_1_I_2a_a_v9 & A & DATA \\
\hline SchD_1_I_2a_b_v9 & $A$ & DATA \\
\hline SchD_1_I_2a_c_V9 & A & DATA \\
\hline SchD_1_I_2a_d_v9 & $\mathrm{F}$ & DATA \\
\hline SchD_1_I_2a_e_v9 & $\mathrm{F}$ & DATA \\
\hline SchD_1_I_2a_f_V9 & $\mathrm{F}$ & DATA \\
\hline SchD_1_I_2a_g_v9 & $\mathrm{F}$ & DATA \\
\hline SchD_1_I_2b & $\mathrm{F}$ & DATA \\
\hline SchD_1_I_2c_a_V1 & A & DATA \\
\hline SchD_1_I_2c_b_V1 & A & DATA \\
\hline
\end{tabular}




\begin{tabular}{|c|c|c|}
\hline \multicolumn{3}{|c|}{ Form sch_d 1 - Table A } \\
\hline Entry Field & Type & Context \\
\hline SchD_1_I_2c_c_V1 & A & DATA \\
\hline SchD_1_I_2c_d_V1 & $\mathrm{F}$ & DATA \\
\hline SchD_1_I_2c_e_V1 & $\mathrm{F}$ & DATA \\
\hline SchD_1_I_2c_f_V1 & $\mathrm{F}$ & DATA \\
\hline SchD_1_I_2c_g_V1 & $\mathrm{F}$ & DATA \\
\hline SchD_1_I_2c_a_V2 & A & DATA \\
\hline SchD_1_I_2c_b_V2 & A & DATA \\
\hline SchD_1_I_2c_c_V2 & A & DATA \\
\hline SchD_1_I_2c_d_V2 & $\mathrm{F}$ & DATA \\
\hline SchD_1_I_2c_e_V2 & $\mathrm{F}$ & DATA \\
\hline SchD_1_I_2c_f_V2 & $\mathrm{F}$ & DATA \\
\hline SchD_1_I_2c_g_V2 & $\mathrm{F}$ & DATA \\
\hline SchD_1_I_2c_a_V3 & A & DATA \\
\hline SchD_1_I_2c_b_V3 & A & DATA \\
\hline SchD_1_I_2c_c_V3 & A & DATA \\
\hline SchD_1_I_2c_d_V3 & $\mathrm{F}$ & DATA \\
\hline SchD_1_I_2c_e_V3 & $\mathrm{F}$ & DATA \\
\hline SchD_1_I_2c_f_V3 & $\mathrm{F}$ & DATA \\
\hline SchD_1_I_2c_g_V3 & $\mathrm{F}$ & DATA \\
\hline SchD_1_I_3 & $\mathrm{F}$ & DATA \\
\hline SchD_1_I_4 & $\mathrm{F}$ & DATA \\
\hline SchD_1_I_5_f & $\mathrm{F}$ & DATA \\
\hline SchD_1_I_5_g & $\mathrm{F}$ & DATA \\
\hline SchD_1_I_6 & $F$ & DATA \\
\hline SchD_1_I_7_f & FU & DATA \\
\hline SchD_1_I_7_g & $\mathrm{F}$ & DATA \\
\hline SchD_1_I_8 & $\mathrm{F}$ & DATA \\
\hline SchD_1_II_9a_a_V1 & A & DATA \\
\hline SchD_1_II_9a_b_V1 & A & DATA \\
\hline SchD_1_II_9a_c_V1 & A & DATA \\
\hline SchD_1_II_9a_d_V1 & $\mathrm{F}$ & DATA \\
\hline SchD_1_II_9a_e_V1 & $\mathrm{F}$ & DATA \\
\hline SchD_1_II_9a_f_V1 & $\mathrm{F}$ & DATA \\
\hline SchD_1_II_9a_g_V1 & $\mathrm{F}$ & DATA \\
\hline SchD_1_II_9a_a_V2 & A & DATA \\
\hline SchD_1_II_9a_b_V2 & A & DATA \\
\hline SchD_1_II_9a_c_V2 & A & DATA \\
\hline
\end{tabular}




\begin{tabular}{|c|c|c|}
\hline \multicolumn{3}{|c|}{ Form sch_d 1 - Table A } \\
\hline Entry Field & Type & Context \\
\hline SchD_1_II_9a_d_V2 & $\mathrm{F}$ & DATA \\
\hline SchD_1_II_9a_e_V2 & $\mathrm{F}$ & DATA \\
\hline SchD_1_II_9a_f_V2 & $\mathrm{F}$ & DATA \\
\hline SchD_1_II_9a_g_V2 & $\mathrm{F}$ & DATA \\
\hline SchD_1_II_9a_a_V3 & A & DATA \\
\hline SchD_1_II_9a_b_V3 & A & DATA \\
\hline SchD_1_II_9a_c_V3 & A & DATA \\
\hline SchD_1_II_9a_d_V3 & $\mathrm{F}$ & DATA \\
\hline SchD_1_II_9a_e_V3 & $\mathrm{F}$ & DATA \\
\hline SchD_1_II_9a_f_V3 & $\mathrm{F}$ & DATA \\
\hline SchD_1_II_9a_g_V3 & $\mathrm{F}$ & DATA \\
\hline SchD_1_II_9a_a_V4 & A & DATA \\
\hline SchD_1_II_9a_b_V4 & A & DATA \\
\hline SchD_1_II_9a_c_V4 & $A$ & DATA \\
\hline SchD_1_II_9a_d_V4 & $\mathrm{F}$ & DATA \\
\hline SchD_1_II_9a_e_V4 & $\mathrm{F}$ & DATA \\
\hline SchD_1_II_9a_f_V4 & $\mathrm{F}$ & DATA \\
\hline SchD_1_II_9a_g_V4 & $\mathrm{F}$ & DATA \\
\hline SchD_1_II_9a_a_V5 & $\mathrm{A}$ & DATA \\
\hline SchD_1_II_9a_b_V5 & A & DATA \\
\hline SchD_1_II_9a_c_V5 & A & DATA \\
\hline SchD_1_II_9a_d_V5 & $\mathrm{F}$ & DATA \\
\hline SchD_1_II_9a_e_V5 & $\mathrm{F}$ & DATA \\
\hline SchD_1_II_9a_f_V5 & $\mathrm{F}$ & DATA \\
\hline SchD_1_II_9a_g_V5 & $\mathrm{F}$ & DATA \\
\hline SchD_1_II_9a_a_V6 & A & DATA \\
\hline SchD_1_II_9a_b_V6 & A & DATA \\
\hline SchD_1_II_9a_c_V6 & A & DATA \\
\hline SchD_1_II_9a_d_V6 & $\mathrm{F}$ & DATA \\
\hline SchD_1_II_9a_e_V6 & $\mathrm{F}$ & DATA \\
\hline SchD_1_II_9a_f_v6 & $\mathrm{F}$ & DATA \\
\hline SchD_1_II_9a_g_V6 & $\mathrm{F}$ & DATA \\
\hline SchD_1_II_9a_a_V7 & A & DATA \\
\hline SchD_1_II_9a_b_V7 & A & DATA \\
\hline SchD_1_II_9a_c_V7 & A & DATA \\
\hline SchD_1_II_9a_d_V7 & $\mathrm{F}$ & DATA \\
\hline SchD_1_II_9a_e_V7 & $\mathrm{F}$ & DATA \\
\hline
\end{tabular}




\begin{tabular}{|c|c|c|}
\hline \multicolumn{3}{|c|}{ Form sch_d 1 - Table A } \\
\hline Entry Field & Type & Context \\
\hline SchD_1_II_9a_f_V7 & $\mathrm{F}$ & DATA \\
\hline SchD_1_II_9a_g_V7 & $\mathrm{F}$ & DATA \\
\hline SchD_1_II_9a_a_V8 & $A$ & DATA \\
\hline SchD_1_II_9a_b_V8 & A & DATA \\
\hline SchD_1_II_9a_c_V8 & A & DATA \\
\hline SchD_1_II_9a_d_V8 & $\mathrm{F}$ & DATA \\
\hline SchD_1_II_9a_e_V8 & $\mathrm{F}$ & DATA \\
\hline SchD_1_II_9a_f_v8 & $\mathrm{F}$ & DATA \\
\hline SchD_1_II_9a_g_V8 & $\mathrm{F}$ & DATA \\
\hline SchD_1_II_9a_a_V9 & A & DATA \\
\hline SchD_1_II_9a_b_v9 & A & DATA \\
\hline SchD_1_II_9a_c_V9 & A & DATA \\
\hline SchD_1_II_9a_d_V9 & $\mathrm{F}$ & DATA \\
\hline SchD_1_II_9a_e_V9 & $\mathrm{F}$ & DATA \\
\hline SchD_1_II_9a_f_V9 & $\mathrm{F}$ & DATA \\
\hline SchD_1_II_9a_g_v9 & $\mathrm{F}$ & DATA \\
\hline SchD_1_II_9b & $\mathrm{F}$ & DATA \\
\hline SchD_1_II_9c_a_V1 & A & DATA \\
\hline SchD_1_II_9c_b_V1 & A & DATA \\
\hline SchD_1_II_9c_c_V1 & A & DATA \\
\hline SchD_1_II_9c_d_V1 & $\mathrm{F}$ & DATA \\
\hline SchD_1_II_9c_e_V1 & $\mathrm{F}$ & DATA \\
\hline SchD_1_II_9c_f_V1 & $\mathrm{F}$ & DATA \\
\hline SchD_1_II_9c_9_V1 & $\mathrm{F}$ & DATA \\
\hline SchD_1_II_9c_a_V2 & A & DATA \\
\hline SchD_1_II_9c_b_V2 & A & DATA \\
\hline SchD_1_II_9c_c_V2 & A & DATA \\
\hline SchD_1_II_9c_d_V2 & $\mathrm{F}$ & DATA \\
\hline SchD_1_II_9c_e_V2 & $\mathrm{F}$ & DATA \\
\hline SchD_1_II_9c_f_V2 & $\mathrm{F}$ & DATA \\
\hline SchD_1_II_9c_g_V2 & $\mathrm{F}$ & DATA \\
\hline SchD_1_II_9c_a_V3 & A & DATA \\
\hline SchD_1_II_9c_b_V3 & A & DATA \\
\hline SchD_1_II_9c_c_V3 & A & DATA \\
\hline SchD_1_II_9c_d_V3 & $\mathrm{F}$ & DATA \\
\hline SchD_1_II_9c_e_V3 & $\mathrm{F}$ & DATA \\
\hline SchD_1_II_9c_f_V3 & $\mathrm{F}$ & DATA \\
\hline
\end{tabular}




\begin{tabular}{|l|c|c|}
\hline \multicolumn{3}{|c|}{ Form sch_d 1 - Table A } \\
\hline \multicolumn{1}{|c|}{ Entry Field } & Type & Context \\
\hline SchD_1_II_9c_g_V3 & F & DATA \\
\hline SchD_1_II_10 & F & DATA \\
\hline SchD_1_II_11 & F & DATA \\
\hline SchD_1_II_12_f & F & DATA \\
\hline SchD_1_II112_g & F & DATA \\
\hline SchD_1_II113 & F & DATA \\
\hline SchD_1_II_14 & F & DATA \\
\hline SchD_1_II_15 & F & DATA \\
\hline SchD_1_II_16_f & FU & DATA \\
\hline SchD_1_II_16_g & F & DATA \\
\hline SchD_1_I_17 & F & DATA \\
\hline
\end{tabular}


Form Schedule d 2- Table A

\begin{tabular}{|l|c|c|}
\hline \multicolumn{3}{|c|}{ Form sch_d 2 - Table A } \\
\hline \multicolumn{1}{|c|}{ Entry Field } & Type & Context \\
\hline SchD_2_L_H1 & A & NAME \\
\hline SchD_2_L_H2 & A & SSN \\
\hline SchD_2_II_18 & F & DATA \\
\hline SchD_2_III19 & FU & DATA \\
\hline SchD_2_IV_20 & F & DATA \\
\hline SchD_2_IV_21 & F & DATA \\
\hline SchD_2_IV_22 & F & DATA \\
\hline SchD_2_IV_23 & F & DATA \\
\hline SchD_2_IV_24 & F & DATA \\
\hline SchD_2_IV_25 & F & DATA \\
\hline SchD_2_IV_26 & F & DATA \\
\hline SchD_2_IV_27 & F & DATA \\
\hline SchD_2_IV_28 & F & DATA \\
\hline SchD_2_IV_29 & F & DATA \\
\hline SchD_2_V_30 & ICON & DATA \\
\hline SchD_2_V_31 & FP & DATA \\
\hline SchD_2_V_32 & FP & DATA \\
\hline SchD_2_VI_33 & F & DATA \\
\hline SchD_2_VI_34 & F & DATA \\
\hline SchD_2_VI_35 & F & DATA \\
\hline SchD_2_VI_36 & F & DATA \\
\hline SchD_2_VI_37 & F & DATA \\
\hline SchD_2_VI_38_V1 & A & DATA \\
\hline SchD_2_VI_38_V2 & CA & DATA \\
\hline SchD_2_VI_38_V3 & CA & DATA \\
\hline SchD_2_VI_38_H1_V4 & FA & DATA \\
\hline SchD_2_VI_38_H2_V4 & F & DATA \\
\hline SchD_2_VI_39 & DATA \\
\hline
\end{tabular}


Form Schedule e 1- Table A

\begin{tabular}{|l|c|c|}
\hline \multicolumn{3}{|c|}{ Form sch_e 1 - Table A } \\
\hline \multicolumn{1}{|c|}{ Entry Field } & Type & Context \\
\hline SchE_1_L_H1 & A & NAME \\
\hline SchE_1_L_H2 & A & SSN \\
\hline SchE_1_1_A & A & DATA \\
\hline SchE_1_2_A_H1 & ICON & DATA \\
\hline SchE_1_2_A_H2 & ICON & DATA \\
\hline SchE_1_3_A_H1 & ICON & DATA \\
\hline SchE_1_3_A_H2 & ICON & DATA \\
\hline SchE_1_1_B & A & DATA \\
\hline SchE_1_2_B_H1 & ICON & DATA \\
\hline SchE_1_2_B_H2 & ICON & DATA \\
\hline SchE_1_3_B_H1 & ICON & DATA \\
\hline SchE_1_3_B_H2 & ICON & DATA \\
\hline SchE_1_1_C & A & DATA \\
\hline SchE_1_2_C_H1 & ICON & DATA \\
\hline SchE_1_2_C_H2 & ICON & DATA \\
\hline SchE_1_3_C_H1 & ICON & DATA \\
\hline SchE_1_3_C_H2 & ICON & DATA \\
\hline SchE_1_4_A & F & DATA \\
\hline SchE_1_4_B & F & DATA \\
\hline SchE_1_4_C & F & DATA \\
\hline SchE_1_4 & F & DATA \\
\hline SchE_1_5_A & F & DATA \\
\hline SchE_1_5_B & F & DATA \\
\hline SchE_1_5_C & F & DATA \\
\hline SchE_1_5 & F & DATA \\
\hline SchE_1_6_A & F & DATA \\
\hline SchE_1_6_B & F & DATA \\
\hline SchE_1_6_C & F & DATA \\
\hline SchE_1_7_A & F & DATA \\
\hline SchE_1_7_B & F & DATA \\
\hline SchE_1_7_C & F & DATA \\
\hline SchE_1_8_A & F & DATA \\
\hline SchE_1_8_B & F & DATA \\
\hline SchE_1_8_C & F & DATA \\
\hline
\end{tabular}




\begin{tabular}{|c|c|c|}
\hline \multicolumn{3}{|c|}{ Form sch_e 1 - Table A } \\
\hline Entry Field & Type & Context \\
\hline SchE_1_9_A & $\mathrm{F}$ & DATA \\
\hline SchE_1_9_B & $\mathrm{F}$ & DATA \\
\hline SchE_1_9_C & $\mathrm{F}$ & DATA \\
\hline SchE_1_10_A & $\mathrm{F}$ & DATA \\
\hline SchE_1_10_B & $\mathrm{F}$ & DATA \\
\hline SchE_1_10_C & $\mathrm{F}$ & DATA \\
\hline SchE_1_11_A & $\mathrm{F}$ & DATA \\
\hline SchE_1_11_B & $\mathrm{F}$ & DATA \\
\hline SchE_1_11_C & $\mathrm{F}$ & DATA \\
\hline SchE_1_12_A & $\mathrm{F}$ & DATA \\
\hline SchE_1_12_B & $\mathrm{F}$ & DATA \\
\hline SchE_1_12_C & $\mathrm{F}$ & DATA \\
\hline SchE_1_12 & $\mathrm{F}$ & DATA \\
\hline SchE_1_13_A & $\mathrm{F}$ & DATA \\
\hline SchE_1_13_B & $\mathrm{F}$ & DATA \\
\hline SchE_1_13_C & $\mathrm{F}$ & DATA \\
\hline SchE_1_14_A & $\mathrm{F}$ & DATA \\
\hline SchE_1_14_B & $\mathrm{F}$ & DATA \\
\hline SchE_1_14_C & $\mathrm{F}$ & DATA \\
\hline SchE_1_15_A & $\mathrm{F}$ & DATA \\
\hline SchE_1_15_B & $\mathrm{F}$ & DATA \\
\hline SchE_1_15_C & $\mathrm{F}$ & DATA \\
\hline SchE_1_16_A & $\mathrm{F}$ & DATA \\
\hline SchE_1_16_B & $\mathrm{F}$ & DATA \\
\hline SchE_1_16_C & $\mathrm{F}$ & DATA \\
\hline SchE_1_17_A & $\mathrm{F}$ & DATA \\
\hline SchE_1_17_B & $\mathrm{F}$ & DATA \\
\hline SchE_1_17_C & $\mathrm{F}$ & DATA \\
\hline SchE_1_18_A & $\mathrm{F}$ & DATA \\
\hline SchE_1_18_B & $\mathrm{F}$ & DATA \\
\hline SchE_1_18_C & $\mathrm{F}$ & DATA \\
\hline SchE_1_19_V1 & A & DATA \\
\hline SchE_1_19_A_V1 & $\mathrm{F}$ & DATA \\
\hline SchE_1_19_B_V1 & $F$ & DATA \\
\hline SchE_1_19_C_V1 & $\mathrm{F}$ & DATA \\
\hline SchE_1_19_V2 & A & DATA \\
\hline SchE_1_19_A_V2 & $\mathrm{F}$ & DATA \\
\hline
\end{tabular}




\begin{tabular}{|l|c|c|}
\hline \multicolumn{3}{|c|}{ Form sch_e 1 - Table A } \\
\hline \multicolumn{1}{|c|}{ Entry Field } & Type & Context \\
\hline SchE_1_19_B_V2 & F & DATA \\
\hline SchE_1_19_C_V2 & F & DATA \\
\hline SchE_1_19_V3 & A & DATA \\
\hline SchE_1_19_A_V3 & F & DATA \\
\hline SchE_1_19_B_V3 & F & DATA \\
\hline SchE_1_19_C_V3 & F & DATA \\
\hline SchE_1_19_V4 & A & DATA \\
\hline SchE_1_19_A_V4 & F & DATA \\
\hline SchE_1_19_B_V4 & F & DATA \\
\hline SchE_1_19_C_V4 & F & DATA \\
\hline SchE_1_19_V5 & A & DATA \\
\hline SchE_1_19_A_V5 & F & DATA \\
\hline SchE_1_19_B_V5 & F & DATA \\
\hline SchE_1_19_C_V5 & F & DATA \\
\hline SchE_1_20_A & F & DATA \\
\hline SchE_1_20_B & F & DATA \\
\hline SchE_1_20_C & F & DATA \\
\hline SchE_1_20 & F & DATA \\
\hline SchE_1_21_A & F & DATA \\
\hline SchE_1_21_B & F & DATA \\
\hline SchE_1_21_C & F & DATA \\
\hline SchE_1_21 & F & DATA \\
\hline SchE_1_22_A & F & DATA \\
\hline SchE_1_22_B & F & DATA \\
\hline SchE_1_22_C & F & DATA \\
\hline SchE_1_23_A & F & DATA \\
\hline SchE_1_23_B & F & DATA \\
\hline SchE_1_23_C & F & DATA \\
\hline SchE_1_24_A & FATA \\
\hline SchE_1_24_B & F & DATA \\
\hline SchE_1_24_C & F & DATA \\
\hline SchE_1_25 & DATA \\
\hline SchE_1_26 & FU & DATA \\
\hline SchE_1_27 & DATA \\
\hline SchE_1_28 & DATA \\
\hline SchE_1_29 &
\end{tabular}




\section{Form Schedule e 2- Table A}

\begin{tabular}{|l|c|c|}
\hline \multicolumn{3}{|c|}{ Form sch_e 2 - Table A } \\
\hline \multicolumn{1}{|c|}{ Ary Field } & Type & Context \\
\hline SchE_2_L_H1 & A & NAME \\
\hline SchE_2_L_H2 & A & SSN \\
\hline SchE_2_II_A_a & A & DATA \\
\hline SchE_2_II_A_b & A & DATA \\
\hline SchE_2_II_A_c & ICON & DATA \\
\hline SchE_2_II_A_d & A & DATA \\
\hline SchE_2_II_A_e & ICON & DATA \\
\hline SchE_2_IIA_f & ICON & DATA \\
\hline SchE_2_II_B_a & A & DATA \\
\hline SchE_2_II_B_b & A & DATA \\
\hline SchE_2_II_B_c & ICON & DATA \\
\hline SchE_2_II_B_d & A & DATA \\
\hline SchE_2_IIB_e & ICON & DATA \\
\hline SchE_2_II_B_f & ICON & DATA \\
\hline SchE_2_IICC_a & A & DATA \\
\hline SchE_2_II_C_b & A & DATA \\
\hline SchE_2_II_C_c & ICON & DATA \\
\hline SchE_2_II_C_d & A & DATA \\
\hline SchE_2_II_C_e & ICON & DATA \\
\hline SchE_2_II_C_f & ICON & DATA \\
\hline SchE_2_IID_a & A & DATA \\
\hline SchE_2_II_D_b & A & DATA \\
\hline SchE_2_IID_c & ICON & DATA \\
\hline SchE_2_II_D_d & A & DATA \\
\hline SchE_2_II_D_e & ICON & DATA \\
\hline SchE_2_IID_f & ICON & DATA \\
\hline SchE_2_II_E_a & A & DATA \\
\hline SchE_2_II_E_b & A & DATA \\
\hline SchE_2_IIE_c & ICON & DATA \\
\hline SchE_2_II_E_d & A & DATA \\
\hline SchE_2_IIE_e & ICON & DATA \\
\hline SchE_2_II_E_f & ICON & DATA \\
\hline SchE_2_II_A_d & FU & DATA \\
\hline & & \\
\hline & & \\
\hline
\end{tabular}




\begin{tabular}{|c|c|c|}
\hline \multicolumn{3}{|c|}{ Form sch_e 2 - Table A } \\
\hline Entry Field & Type & Context \\
\hline SchE_2_II_A_h & $\mathrm{F}$ & DATA \\
\hline SchE_2_II_A_i & FU & DATA \\
\hline SchE_2_II_A_j & $\mathrm{FU}$ & DATA \\
\hline SchE_2_II_A_k & $\mathrm{F}$ & DATA \\
\hline SchE_2_II_B_g & FU & DATA \\
\hline SchE_2_II_B_h & $\mathrm{F}$ & DATA \\
\hline SchE_2_II_B_i & $\mathrm{FU}$ & DATA \\
\hline SchE_2_II_B_j & FU & DATA \\
\hline SchE_2_II_B_k & $\mathrm{F}$ & DATA \\
\hline SchE_2_II_C_g & FU & DATA \\
\hline SchE_2_II_C_h & $\mathrm{F}$ & DATA \\
\hline SchE_2_II_C_i & FU & DATA \\
\hline SchE_2_II_C_j & FU & DATA \\
\hline SchE_2_II_C_k & $\mathrm{F}$ & DATA \\
\hline SchE_2_II_D_g & $\mathrm{FU}$ & DATA \\
\hline SchE_2_II_D_h & $\mathrm{F}$ & DATA \\
\hline SchE_2_II_D_i & FU & DATA \\
\hline SchE_2_II_D_j & $\mathrm{FU}$ & DATA \\
\hline SchE_2_II_D_k & $\mathrm{F}$ & DATA \\
\hline SchE_2_II_E_g & FU & DATA \\
\hline SchE_2_II_E_h & $\mathrm{F}$ & DATA \\
\hline SchE_2_II_E_i & FU & DATA \\
\hline SchE_2_II_E_j & $\mathrm{FU}$ & DATA \\
\hline SchE_2_II_E_k & $\mathrm{F}$ & DATA \\
\hline SchE_2_II_30a_h & $\mathrm{F}$ & DATA \\
\hline SchE_2_II_30a_k & $\mathrm{F}$ & DATA \\
\hline SchE_2_II_30b_g & $\mathrm{FU}$ & DATA \\
\hline SchE_2_II_30b_i & FU & DATA \\
\hline SchE_2_II_30b_j & FU & DATA \\
\hline SchE_2_II_31 & $\mathrm{F}$ & DATA \\
\hline SchE_2_II_32 & FU & DATA \\
\hline SchE_2_II_33 & $\mathrm{F}$ & DATA \\
\hline SchE_2_III_A_a & A & DATA \\
\hline SchE_2_III_A_b & A & DATA \\
\hline SchE_2_III_B_a & A & DATA \\
\hline SchE_2_III_B_b & A & DATA \\
\hline SchE_2_III_C_a & A & DATA \\
\hline
\end{tabular}




\begin{tabular}{|c|c|c|}
\hline \multicolumn{3}{|c|}{ Form sch_e 2 - Table A } \\
\hline Entry Field & Type & Context \\
\hline SchE_2_III_C_b & A & DATA \\
\hline SchE_2_III_A_c & FU & DATA \\
\hline SchE_2_III_A_d & $F$ & DATA \\
\hline SchE_2_III_A_e & FU & DATA \\
\hline SchE_2_III_A_f & $\mathrm{F}$ & DATA \\
\hline SchE_2_III_B_C & FU & DATA \\
\hline SchE_2_III_B_d & $F$ & DATA \\
\hline SchE_2_III_B_e & FU & DATA \\
\hline SchE_2_III_B_f & $\mathrm{F}$ & DATA \\
\hline SchE_2_III_C_c & FU & DATA \\
\hline SchE_2_III_C_d & $\mathrm{F}$ & DATA \\
\hline SchE_2_III_C_e & FU & DATA \\
\hline SchE_2_III_C_f & $\mathrm{F}$ & DATA \\
\hline SchE_2_III_34a_d & $\mathrm{F}$ & DATA \\
\hline SchE_2_III_34a_f & $\mathrm{F}$ & DATA \\
\hline SchE_2_III_34b_c & $\mathrm{FU}$ & DATA \\
\hline SchE_2_III_34b_e & $\mathrm{FU}$ & DATA \\
\hline SchE_2_III_35 & $\mathrm{F}$ & DATA \\
\hline SchE_2_III_36 & $\mathrm{FU}$ & DATA \\
\hline SchE_2_III_37 & $F$ & DATA \\
\hline SchE_2_IV_a & A & DATA \\
\hline SchE_2_IV_b & A & DATA \\
\hline SchE_2_IV_c & $\mathrm{F}$ & DATA \\
\hline SchE_2_IV_d & $\mathrm{F}$ & DATA \\
\hline SchE_2_IV_e & $\mathrm{F}$ & DATA \\
\hline SchE_2_IV_38 & $\mathrm{F}$ & DATA \\
\hline SchE_2_V_39 & $F$ & DATA \\
\hline SchE_2_V_40 & $\mathrm{FU}$ & DATA \\
\hline SchE_2_V_41 & $F$ & DATA \\
\hline SchE_2_VI_42 & $\mathrm{F}$ & DATA \\
\hline SchE_2_VI_43 & $\mathrm{F}$ & DATA \\
\hline
\end{tabular}




\section{Form Schedule f 1- Table A}

\begin{tabular}{|l|c|c|}
\hline \multicolumn{3}{|c|}{ Form sch_f 1 - Table A } \\
\hline \multicolumn{1}{|c|}{ Entry Field } & Type & Context \\
\hline SchF_1_L_H1 & A & NAME \\
\hline SchF_1_L_H2 & A & SSN \\
\hline SchF_1_A & A & DATA \\
\hline SchF_1_B & A & DATA \\
\hline SchF_1_C_H1 & ICON & DATA \\
\hline SchF_1_C_H2 & ICON & DATA \\
\hline SchF_1_D & A & DATA \\
\hline SchF_1_E_H1 & ICON & DATA \\
\hline SchF_1_E_H2 & ICON & DATA \\
\hline SchF_1_F_H1 & ICON & DATA \\
\hline SchF_1_F_H2 & ICON & DATA \\
\hline SchF_1_G_H1 & ICON & DATA \\
\hline SchF_1_G_H2 & ICON & DATA \\
\hline SchF_1_G_H3 & ICON & DATA \\
\hline SchF_1_I_1 & F & DATA \\
\hline SchF_1_I_2 & F & DATA \\
\hline SchF_1_I_3 & F & DATA \\
\hline SchF_1_I_4 & F & DATA \\
\hline SchF_1_I_5a & F & DATA \\
\hline SchF_1_I_5b & F & DATA \\
\hline SchF_1_I_6 & F & DATA \\
\hline SchF_1_I_7a & F & DATA \\
\hline SchF_1_I_7b & F & DATA \\
\hline SchF_1_I_8a & F & DATA \\
\hline SchF_1_I_8b & F & DATA \\
\hline SchF_1_I_8c & F & DATA \\
\hline SchF_1_I_9a & F & DATA \\
\hline SchF_1_I_9b & F & DATA \\
\hline SchF_1_I_9c & F & DATA \\
\hline SchF_1_I_9d & F & DATA \\
\hline SchF_1_I_10 & DATA \\
\hline SchF_1_I_11 & F & DATA \\
\hline SchF_1_I_12 & F & DATA \\
\hline SchF_1_I_13 & DATA \\
\hline
\end{tabular}




\begin{tabular}{|c|c|c|}
\hline \multicolumn{3}{|c|}{ Form sch_f 1 - Table A } \\
\hline Entry Field & Type & Context \\
\hline SchF_1_II_14 & $\mathrm{F}$ & DATA \\
\hline SchF_1_II_15 & $F$ & DATA \\
\hline SchF_1_II_16 & $\mathrm{F}$ & DATA \\
\hline SchF_1_II_17 & $\mathrm{F}$ & DATA \\
\hline SchF_1_II_18 & $\mathrm{F}$ & DATA \\
\hline SchF_1_II_19 & $\mathrm{F}$ & DATA \\
\hline SchF_1_II_20 & $\mathrm{F}$ & DATA \\
\hline SchF_1_II_21 & $\mathrm{F}$ & DATA \\
\hline SchF_1_II_22 & $\mathrm{F}$ & DATA \\
\hline SchF_1_II_23 & $\mathrm{F}$ & DATA \\
\hline SchF_1_II_24a & $\mathrm{F}$ & DATA \\
\hline SchF_1_II_24b & $\mathrm{F}$ & DATA \\
\hline SchF_1_II_25a & $\mathrm{F}$ & DATA \\
\hline SchF_1_II_25b & $\mathrm{F}$ & DATA \\
\hline SchF_1_II_25c & $\mathrm{F}$ & DATA \\
\hline SchF_1_II_26 & $\mathrm{F}$ & DATA \\
\hline SchF_1_II_27 & $\mathrm{F}$ & DATA \\
\hline SchF_1_II_28 & $\mathrm{F}$ & DATA \\
\hline SchF_1_II_29 & $\mathrm{F}$ & DATA \\
\hline SchF_1_II_30 & $\mathrm{F}$ & DATA \\
\hline SchF_1_II_31 & $\mathrm{F}$ & DATA \\
\hline SchF_1_II_32 & $\mathrm{F}$ & DATA \\
\hline SchF_1_II_33 & $\mathrm{F}$ & DATA \\
\hline SchF_1_II_34 & $\mathrm{F}$ & DATA \\
\hline SchF_1_II_35a_H1 & A & DATA \\
\hline SchF_1_II_35a_H2 & $\mathrm{F}$ & DATA \\
\hline SchF_1_II_35b_H1 & A & DATA \\
\hline SchF_1_II_35b_H2 & $\mathrm{F}$ & DATA \\
\hline SchF_1_II_35c_H1 & A & DATA \\
\hline SchF_1_II_35c_H2 & $\mathrm{F}$ & DATA \\
\hline SchF_1_II_35d_H1 & A & DATA \\
\hline SchF_1_II_35d_H2 & $\mathrm{F}$ & DATA \\
\hline SchF_1_II_35e_H1 & A & DATA \\
\hline SchF_1_II_35e_H2 & $\mathrm{F}$ & DATA \\
\hline SchF_1_II_36 & $\mathrm{F}$ & DATA \\
\hline SchF_1_II_37 & $\mathrm{F}$ & DATA \\
\hline SchF_1_II_38 & $\mathrm{F}$ & DATA \\
\hline
\end{tabular}




\begin{tabular}{|l|c|c|}
\hline \multicolumn{3}{|c|}{ Form sch_f 1 - Table A } \\
\hline \multicolumn{1}{|c|}{ Entry Field } & Type & Context \\
\hline SchF_1_II39 & F & DATA \\
\hline SchF_1_II_40a & ICON & DATA \\
\hline SchF_1_II440b & ICON & DATA \\
\hline
\end{tabular}


Form Schedule f 2- Table A

\begin{tabular}{|l|l|r|}
\hline \multicolumn{3}{|c|}{ Form sch_f 2 - Table A } \\
\hline EchF_y_41 Field & Type & Context \\
\hline SchF_2_42a & F & DATA \\
\hline SchF_2_42b & F & DATA \\
\hline SchF_2_43 & F & DATA \\
\hline SchF_2_44a & F & DATA \\
\hline SchF_2_44b & F & DATA \\
\hline SchF_2_45a & F & DATA \\
\hline SchF_2_45b & F & DATA \\
\hline SchF_2_45c & F & DATA \\
\hline SchF_2_46 & F & DATA \\
\hline SchF_2_47 & F & DATA \\
\hline SchF_2_48 & F & DATA \\
\hline SchF_2_49 & F & DATA \\
\hline SchF_2_50 & F & DATA \\
\hline SchF_2_51 & F & DATA \\
\hline SchF_2_52 & F & DATA \\
\hline SchF_2_53 & F & DATA \\
\hline SchF_2_54 & F & DATA \\
\hline SchF_2_55 & F & DATA \\
\hline
\end{tabular}


Form Schedule se 1 - Table A

\begin{tabular}{|l|c|c|}
\hline \multicolumn{3}{|c|}{ Form sch_se 1 - Table A } \\
\hline \multicolumn{1}{|c|}{ Entry Field } & Type & Context \\
\hline SchSE_1_L_H1 & A & NAME \\
\hline SchSE_1_L_H2 & A & SSN \\
\hline SchSE_1_1 & F & DATA \\
\hline SchSE_1_2 & F & DATA \\
\hline SchSE_1_3 & F & DATA \\
\hline SchSE_1_5 & F & DATA \\
\hline SchSE_1_6 & F & DATA \\
\hline SchSE_1_7 & F & DATA \\
\hline SchSE_1_8 & F & DATA \\
\hline
\end{tabular}




\section{Form Schedule se 2 - Table A}

\begin{tabular}{|l|c|c|}
\hline \multicolumn{3}{|c|}{ Form sch_se 2 - Table A } \\
\hline \multicolumn{1}{|c|}{ Entry Field } & Type & Context \\
\hline SchSE_2_L_H1 & A & NAME \\
\hline SchSE_2_L_H2 & A & SSN \\
\hline SchSE_2_A_ & ICON & DATA \\
\hline SchSE_2_I_1 & F & DATA \\
\hline SchSE_2_I_2 & F & DATA \\
\hline SchSE_2_I_3a & F & DATA \\
\hline SchSE_2_I_3b & F & DATA \\
\hline SchSE_2_I_3c & F & DATA \\
\hline SchSE_2_I_5a & F & DATA \\
\hline SchSE_2_I_5b & F & DATA \\
\hline SchSE_2_I_5c & F & DATA \\
\hline SchSE_2_I_6a & F & DATA \\
\hline SchSE_2_I_6b & F & DATA \\
\hline SchSE_2_I_6c & F & DATA \\
\hline SchSE_2_I_6d & F & DATA \\
\hline SchSE_2_I_7 & F & DATA \\
\hline SchSE_2_I_8 & F & DATA \\
\hline SchSE_2_II_10 & F & DATA \\
\hline SchSE_2_I_11 & F & DATA \\
\hline SchSE_2_II12 & & DATA \\
\hline
\end{tabular}

